\title{
A textbook example of ram-pressure stripping in the Hydra A/A780 cluster
}

\author{
S. De Grandi ${ }^{1}$, D. Eckert ${ }^{2}, 3$, S. Molendi ${ }^{3}$, M. Girardi ${ }^{4,5}$, E. Roediger ${ }^{6}$, M. Gaspari $^{7, \star}$, F. Gastaldello $^{3}$, S. Ghizzardi ${ }^{3}$, \\ M. Nonino ${ }^{4}$, and M. Rossetti ${ }^{3,8}$
}

1 INAF-Osservatorio Astronomico di Brera, via E. Bianchi 46, 23807 Merate, Italy e-mail: sabrina.degrandi@brera.inaf.it

2 Department of Astronomy, University of Geneva, ch. d'Ecogia 16, 1290 Versoix, Switzerland

3 INAF-IASF-Milano, via E. Bassini 15, 20133 Milano, Italy

4 Dipartimento di Fisica dell'Università degli Studi di Trieste, Sezione di Astronomia, via Tiepolo 11, 34143 Trieste, Italy

5 INAF-Osservatorio Astronomico di Trieste, via Tiepolo 11, 34143 Trieste, Italy

6 E.A. Milne Centre for Astrophysics, Department of Physics \& Mathematics, University of Hull, Hull, HU6 7RX, UK

7 Department of Astrophysical Sciences, Princeton University, Princeton, NJ 08544, USA

8 Università degli Studi Milano, via Celoria 16, 20133 Milano, Italy

Received 30 May 2015 / Accepted 18 February 2016

\begin{abstract}
In the current epoch, one of the main mechanisms driving the growth of galaxy clusters is the continuous accretion of group-scale halos. In this process, the ram pressure applied by the hot intracluster medium on the gas content of the infalling group is responsible for stripping the gas from its dark-matter halo, which gradually leads to the virialisation of the infalling gas in the potential well of the main cluster. Using deep wide-field observations of the poor cluster Hydra A/A780 with XMM-Newton and Suzaku, we report on the discovery of an infalling galaxy group $1.1 \mathrm{Mpc}$ south of the cluster core. The presence of a substructure is confirmed by a dynamical study of the galaxies in this region. A wake of stripped gas is trailing behind the group over a projected scale of $760 \mathrm{kpc}$. The temperature of the gas along the wake is constant at $k T \sim 1.3 \mathrm{keV}$, which is about a factor of two less than the temperature of the surrounding plasma. We observe a cold front pointing westwards compared to the peak of the group, which indicates that the group is currently not moving in the direction of the main cluster, but is moving along an almost circular orbit. The overall morphology of the group bears remarkable similarities with high-resolution numerical simulations of such structures, which greatly strengthens our understanding of the ram-pressure stripping process.
\end{abstract}

Key words. X-rays: galaxies: clusters - galaxies: clusters: intracluster medium - galaxies: groups: general large-scale structure of Universe

\section{Introduction}

Gravitationally bound structures in the Universe are thought to grow hierarchically through the merging and accretion of smaller structures throughout cosmic time, until they form the most massive galaxy clusters we see today (e.g. Springel et al. 2006). In the outer regions of galaxy clusters, we can observe the accretion of galaxies and groups of galaxies onto the main dark-matter halo, and they contribute significantly to the growth of galaxy clusters in mass, member galaxies, and hot gas (e.g. Berrier et al. 2009; Genel et al. 2010; De Lucia et al. 2012). While the merging processes of two or more massive entities are well studied (Markevitch \& Vikhlinin 2007; Owers et al. 2009, and references therein), the processes leading to the gentle accretion of smaller halos (group scale) onto massive clusters, which represent the main channel ( 70\%, Berrier et al. 2009) of cosmic structure growth, have been more elusive. During infall, the ram pressure applied by the ambient intracluster medium (ICM) is responsible for stripping the gas from its original halo and heating it up (e.g. Gunn \& Gott 1972; Kawata \& Mulchaey 2008; McCarthy et al. 2008), leading to the virialisation of the gas in the main dark-matter halo.

\footnotetext{
^ Einstein and Spitzer Fellow
}

This picture is confirmed by observations of X-ray (e.g. Sun \& Vikhlinin 2005; Machacek et al. 2006; Sun et al. 2007; Randall et al. 2008; Zhang et al. 2013) and atomic (HI) and molecular galaxy wakes behind individual infalling galaxies in the nearest galaxy clusters (e.g. Boselli et al. 2014; Abramson et al. 2011; Sun et al. 2007; Chung et al. 2007). Observations of such features are of prime importance for our understanding of the mechanisms leading to the build-up of galaxy clusters and provide a striking confirmation of the hierarchical scenario of structure formation. The $\Lambda \mathrm{CDM}$ paradigm predicts that at the present epoch there should be approximately one accreting group with a gravitational mass in the range of a few $10^{13} M_{\odot}$ per massive cluster and per Gyr (Dolag et al. 2009). There are a few examples of accreting groups that are directly observed in X-rays, such as A2142 (Eckert et al. 2014), NGC 4389 in Coma (Neumann et al. 2003), and the southern group in A85 (Kempner et al. 2002; Durret et al. 2005; Ichinohe et al. 2015).

In addition to the stripping properties, these features are valuable for the study of ICM physics. Indeed, the survival of the stripped gas in contact with the surrounding ICM can tell us about the conduction timescale in the medium, which is usually found to be much longer than expected from pure Spitzer 
conductivity (Eckert et al. 2014; Sanders et al. 2013). Additionally, Roediger et al. (2015a,b) used high-resolution simulations to study the properties of infalling galaxies, varying the viscosity of the ICM plasma. It was found that the morphology of the tails strongly depends on the viscosity of the fluid: while in the inviscid case Kelvin-Helmholtz $(\mathrm{KH})$ instabilities rapidly develop and induce a fast mixing of the plasma, a high viscosity suppresses $\mathrm{KH}$ instabilities, which results in long, X-ray bright tails.

In 2012 we started an XMM-Newton programme to look for infalling gas clumps in two nearby clusters, A2142 and Hydra A (A780; Abell et al. 1989). This programme allowed us to discover a striking example of infalling substructures in A2142. Specifically, we discovered in this cluster a galaxy group with a mass of a few $10^{13} M_{\odot}$ that appears to be almost completely stripped from its hot gas (Eckert et al. 2014), which constitutes a spectacular tail extending over $800 \mathrm{kpc}$ and is the longest rampressure-stripped tail observed so far. In this paper we focus our study on the outskirts of the other observed cluster, Hydra A, where we discovered another accreting galaxy group, centred on the galaxy LEDA 87445 (Smith et al. 2004) 17' south-east from the centre of Hydra A cluster. This infalling group shows a long low surface-brightness tail (up to $760 \mathrm{kpc}$ ) and a density contact discontinuity (merger cold front) in the opposite direction from the tail, which give important indications about its motion and ram-pressure stripping properties. We complement the $X M M-N e w t o n$ data with a deep Suzaku observation of Hydra A and optical data from the literature.

This paper is structured as follows: in Sect. 2 we describe our XMM-Newton (Sect. 2.1) and Suzaku (Sect. 2.2) data sets together with their respective data reduction, imaging, and spectral analysis techniques. In Sect. 3, we describe the results of the data analysis, concentrating on the description of the morphology (Sect. 3.1) and spectral properties of the new X-ray infalling group (Sect. 3.2). In Sect. 3.3, we analyse the available optical data from the literature for the Hydra A cluster galaxies and quantify the dynamical properties of the group. In Sect. 4, we interpret and discuss our results: thermodynamic and ram-pressure stripping properties of the gas removed from the group are derived in Sects. 4.2-4.4. In Sect. 4.5 we estimate the orbit of the group and in Sect. 4.6 we compare our findings with recent numerical simulations. We summarize our main results in Sect. 5 .

Throughout the paper, we assume a $\Lambda$ CDM cosmology with $H_{\mathrm{o}}=70 \mathrm{~km} \mathrm{~s}^{-1}, \Omega_{\mathrm{m}}=0.3$, and $\Omega_{\Lambda}=0.7$. At the redshift of Hydra A $(z=0.0539)$, this corresponds to $1^{\prime \prime}=1.05 \mathrm{kpc}$. The average temperature of this cluster is $\sim 3.5 \mathrm{keV}$ (David et al. 2001); Sato et al. (2012) derived R500 ${ }^{1}=811 \pm 10 \mathrm{kpc}(\sim 13 \mathrm{ar}-$ cmin) and $\mathrm{R} 200=1189 \pm 96 \mathrm{kpc}(\sim 20 \mathrm{arcmin})$ from the fitting of the Hydra A hydrostatic mass with the Navarro-Frenk-White (Navarro et al. 1996) universal mass profile. All the quoted errors hereafter are at the $1 \sigma$ level.

\section{Data analysis}

\subsection{XMM-Newton}

\subsubsection{Data reduction}

Hydra A/Abell $780(z=0.0539)$ is a cool-core cluster with a virial temperature of $\sim 3.5 \mathrm{keV}$ and a total flux of $2.7 \times 10^{-11} \mathrm{erg} \mathrm{cm}^{-2} \mathrm{~s}^{-1}(0.5-2.0 \mathrm{keV}$ band). Except for cavities in the central regions caused by a giant AGN outburst (McNamara et al. 2000), its X-ray morphology is relaxed.

\footnotetext{
1 For a given overdensity $\Delta, R_{\Delta}$ is the radius for which $M_{\Delta} /\left(4 / 3 \pi R_{\Delta}^{3}\right)=$ $\Delta \rho_{\mathrm{c}}$.
}

Hydra A was extensively observed with XMM-Newton both on-axis (for a total nominal exposure time of $\sim 160 \mathrm{ks}$, Obs. ID 0109980301-501, 0504260101) and off-axis (six pointings along different directions, $\sim 280 \mathrm{ks}$ total; PI: Eckert, Obs. ID. 0694440301-401-701-801, 0725240101, 0761550101), so we obtained a mosaic of this cluster covering the entire azimuth out to the virial radius. We processed the observations using the ESAS tasks as provided in SAS v.13.5. In particular, the southern region of Hydra A was first observed with XMM-Newton on June 2012 for 40.8 ks (PI: Eckert, Obs. ID. 0694440301), on November 2013, for 31 ks (PI: Eckert, Obs. ID. 0725240101), and on May 2015, a subsequent 123.8 ks observation was performed in C time (PI: Eckert, Obs. ID. 0761550101). Since the first observation was badly affected by soft proton flares, we focus exclusively on the second and third observations. After soft protons cleaning procedure (with the mos-filter and pn-filter ESAS tasks; Snowden et al. 2008), the total available clean exposure time for the southern region of Hydra A is $82 \mathrm{ks}$ for MOS1, $88 \mathrm{ks}$ for MOS2, and $27 \mathrm{ks}$ for pn.

\subsubsection{Imaging}

Following Eckert et al. (2014), we extracted count maps in the energy range $0.7-1.2 \mathrm{keV}$ (where the signal-to-background ratio is the highest) for the three EPIC detectors and co-added them to obtain a total EPIC image. To correct the images for vignetting, we extracted individual exposure maps using the XMMSAS task eexpmap and created a total exposure map by summing the individual exposure maps, weighted by the relative effective area of each instrument. Following Snowden et al. (2008), we used a collection of closed-filter observations to produce a map of the non-X-ray background, which we rescaled to our observations by comparing the count rates in the unexposed corners of the field of view. Finally, we created a vignetting-corrected, NXBsubtracted sky image by subtracting the NXB image from the count map and dividing by the exposure map. The resulting image was then smoothed using the XMMSAS adaptive-smoothing tool asmooth, ensuring a target signal to noise of 5 . The final image of Hydra A is shown in Fig. 1; the newly discovered group is peaked on the galaxy LEDA 87445 and is shown in the figure by the white square.

\subsubsection{Spectral analysis}

We performed a spectral analysis of the galaxy group using the two good observations of the SE region in Hydra A (Obs. ID. 0725240101 and 0761550101). Spectra and response files for each region were extracted using the ESAS tasks mos-spectra and pn-spectra from the two observations separately. The spectra of each detector were then summed up using the mathpha, addarf, and addrmf ftools v.6.16. Finally, the total spectra were fit in XSPEC v12.8.1. Since the surface brightness of the group, apart from the emission peak, barely exceeds the background level, we preferred to model the background instead of subtracting it (e.g. Leccardi \& Molendi 2008b). This method requires a careful characterisation of all the various background components to obtain reliable measurements of the relevant parameters. We adopted the following approach to model the different spectral components:

- The non X-ray background (NXB): We used closed-filter observations to estimate the spectrum of the NXB component in each region (ESAS tasks mos-back and pn-back), following the procedure described in Snowden et al. (2008). We approximated the NXB component by a phenomenological 


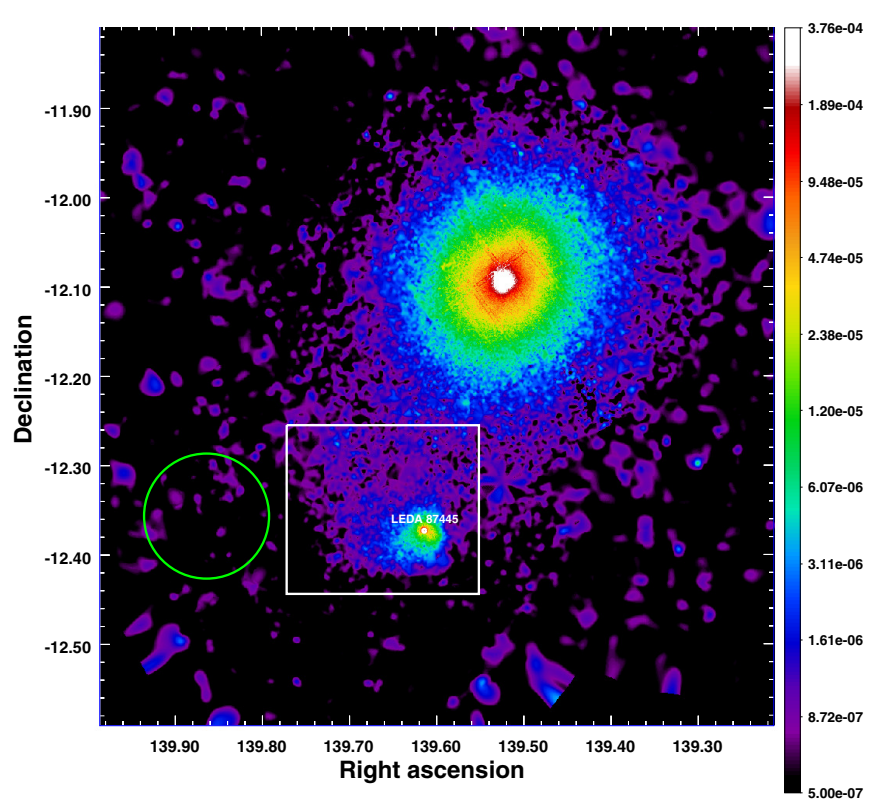

Fig. 1. Adaptively-smoothed, vignetting-corrected XMM/EPIC mosaic image of the Hydra A cluster in the [0.7-1.2] keV band. The group located around the galaxy LEDA 87445 is shown by the white square. The green circle shows the region used to estimate the local background components.

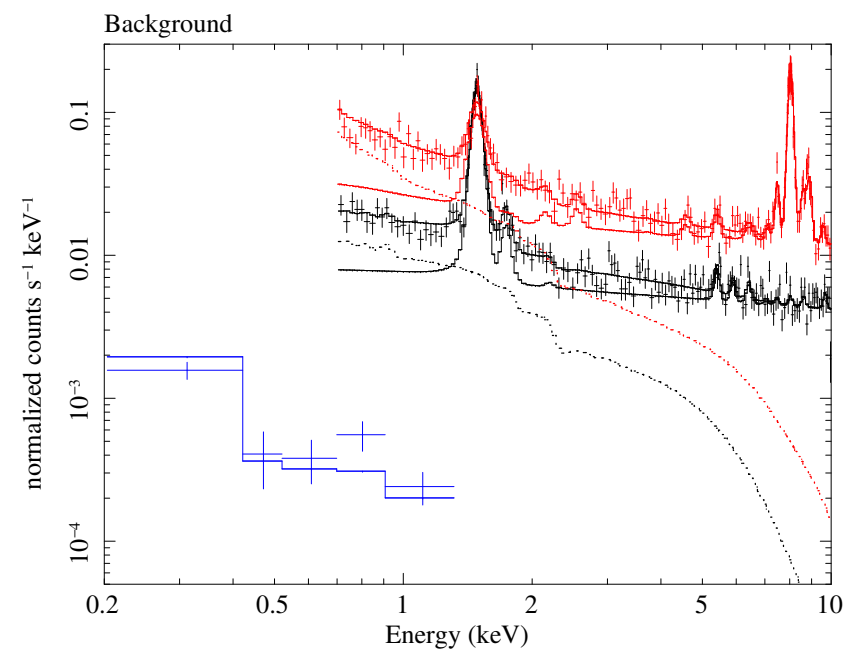

Fig. 2. Spectrum of the background region located at 25.5 arcmin SE of the cluster core used to measure the sky background components from the EPIC pn (red), MOS2 (black; MOS1 was omitted for clarity), and from the ROSAT/PSPC (blue). The solid lines show the NXB model for each instrument, while the dotted lines show the sky background model.

model, which we then included as an additive component in the spectral modelling (Leccardi \& Molendi 2008b). The NXB components of the two MOS detectors are reproduced well by the models in Leccardi \& Molendi (2008b), whereas the NXB spectrum of the pn is best fitted by a double broken power-law model with photon indices fixed to 0.61 (below $1.68 \mathrm{keV}$ ), 0.31 (between 1.68 and $4.09 \mathrm{keV}$ ), and 0.18 (above $4.09 \mathrm{keV}$ ). We left the normalisation of the NXB component free to vary during the fitting procedure, which allows for possible systematic variations of the NXB level. The normalisation of the prominent fluorescence emission lines was also left free. The MOS observations are rather contaminated by quiescent soft protons (QSP) that survived the filtering process (mean IN over OUT ratio of 1.32; De Luca \& Molendi 2004), hence we included a broken power-law component with break energy at $5.0 \mathrm{keV}$, and slopes fixed to 0.4 (below $5 \mathrm{keV}$ ) and 0.8 (above $5 \mathrm{keV}$ ) (Leccardi \& Molendi 2008b) with the normalisation left free to vary. We did not include this component in the analysis of the pn spectra, since the pn observation is only weakly contaminated by residual soft protons (IN over OUT ratio of 1.08).

- The sky background components: we used an offset region located $\sim 25.5$ arcmin away from the cluster core (see circle in Fig. 1), where no cluster emission is detected, to measure the sky background components in the region of Hydra A close to the accreting group. To aid in constraining these components, we added to the EPIC data the ROSAT all-sky survey (RASS) background spectrum, which is sensitive to the 0.1-2.4 keV X-ray regime, and fitted the XMMNewton and RASS data jointly (Snowden et al. 1997). The RASS spectrum was obtained through the HEASARC X-ray background tool in a circular region of 0.15 degree radius overlapping the offset region used for the EPIC data. The sky background was modelled with three-components: i) a power law with photon index fixed to 1.46 to model the cosmic X-ray background (CXB; De Luca \& Molendi 2004); ii) a thermal component at a temperature of $0.22 \mathrm{keV}$ to account for the Galactic halo emission; and iii) an unabsorbed thermal component at $0.11 \mathrm{keV}$ for the local hot bubble. We fixed the CXB normalisation to the value taken from De Luca \& Molendi (2004) rescaled appropriately whereas we left the normalisation s of the other soft components free to vary. The normalisation $s$ for the three background components are $(1.18 \pm 0.20) \times 10^{-6} \mathrm{arcmin}^{-2}$ (Local Bubble), $(7.15 \pm 5.47) \times 10^{-7} \operatorname{arcmin}^{-2}$ (Galactic Halo), and $9.81 \times 10^{-7} \operatorname{arcmin}^{-2}(\mathrm{CXB})$. For the APEC (Local Bubble and Galactic halo), these normalisation $\mathrm{s}$ are expressed in units of $10^{-14} /\left(4 \pi d_{\mathrm{A}}^{2}(1+z)^{2}\right) \int n_{\mathrm{e}} n_{\mathrm{H}} \mathrm{d} V$; for the power law $(\mathrm{CXB})$, this is expressed in units of photons $\mathrm{keV}^{-1} \mathrm{~cm}^{-2} \mathrm{~s}^{-1}$ at $1 \mathrm{keV}$. The best-fit spectrum for the offset region is shown in Fig. 2. As a check we also left the CXB normalisation free to vary in our model. We found that its best-fit value, $(9.46 \pm 1.08) \times 10^{-7} \mathrm{arcmin}^{-2}$, agrees well within the statistical uncertainties with the expected value from De Luca \& Molendi (2004). To model the sky background in a different region, the normalisation of each component was rescaled by the ratio of the corresponding areas, accounting for CCD gaps and bad pixels.

- The source: we modelled the diffuse source emission in each region using the thin-plasma emission code APEC (Smith et al. 2001), leaving temperature, metal abundance, and normalisation as free parameters (the solar abundances were taken from Anders \& Grevesse 1989) and the redshift was fixed to the optical value of the LEDA 87445 galaxy ( $z=0.0548$, see Sect. 3.3). This component is absorbed by the Galactic hydrogen column density along the line of sight, which we fix to the $21 \mathrm{~cm}$ value $\left(N_{\mathrm{H}}=4.54 \times 10^{20} \mathrm{~cm}^{-2}\right.$; Kaberla et al. 2005).

The spectral energy range considered is $0.7-10.0 \mathrm{keV}$. We always used in the spectral fitting procedure the $\mathrm{C}$-statistics rather than the $\chi^{2}$ (Cash 1979), which is preferable for low signal to noise spectra (Nousek \& Shue 1989). To apply the Cash statistic, we performed a minimal grouping of the spectra to avoid channels with no counts. 


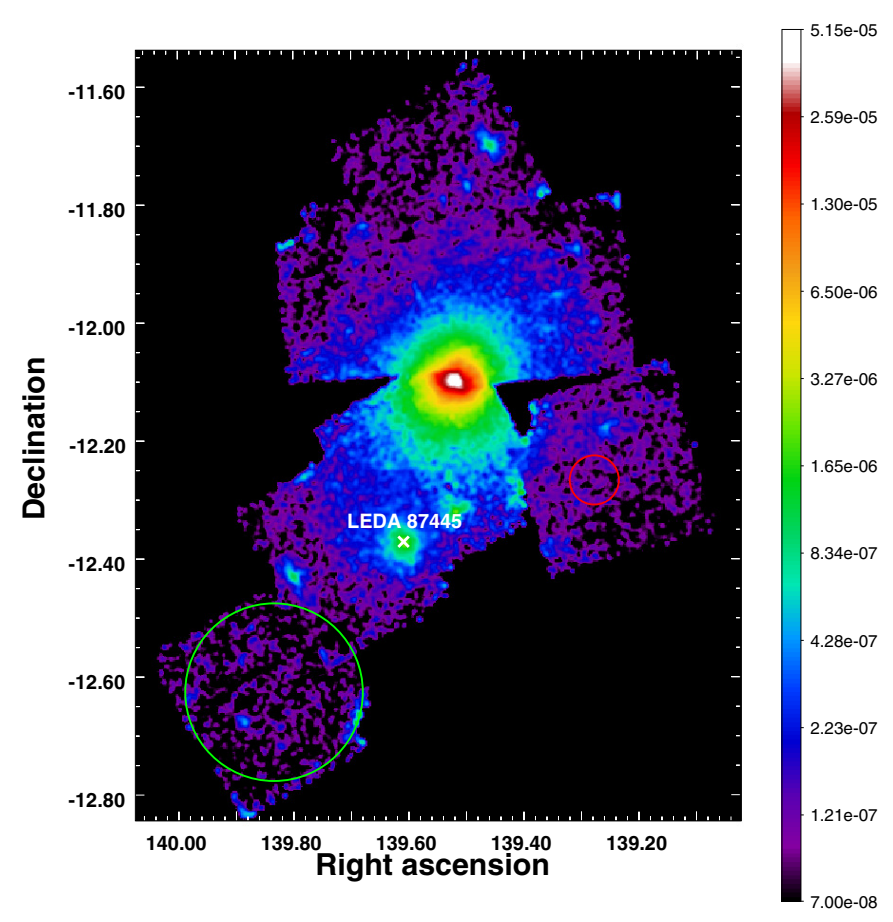

Fig. 3. Exposure-corrected Suzaku/XIS mosaic image of Hydra A combining all seven available pointings. The LEDA 87445 group is highlighted in white. The green circle indicates the background region on the off-source pointing used for this analysis. The red circle is a region used to measure the ICM temperature of Hydra A; details are given in Sect. 3.2.

\subsection{Suzaku}

\subsubsection{Data reduction}

Hydra A was observed by Suzaku in 2012 for a total of $160 \mathrm{ks}$ (PI: Eckert, ID 807087-807091) in addition to two pre-existing Suzaku pointings (70 ks, ID 805007-8; see Sato et al. 2012). This corresponds to a total of six pointings covering the entire azimuth of the cluster out to $R_{200}$, plus an off-source pointing located $\sim 40$ arcmin from the cluster core. We processed all the observations using the Suzaku FTOOLS as provided in HEAsoft v6.12 and the Suzaku CALDB v20130305. We reprocessed the event files using the tool aepipeline, restricting to observing periods with cut-off rigidity $\mathrm{COR}>6$. Suzaku/XIS events obtained in $3 \times 3$ and $5 \times 5$ editing modes were combined using Xselect.

\subsubsection{Imaging analysis}

For each observation, we extracted photon images in the [0.52] keV band for the two front-illuminated chips (XIS0 and XIS3) and for the back-illuminated CCD (XIS1) and created exposure maps using the xisexpmapgen tool. We then produced an exposure-corrected mosaic image by combining all three detectors and all observations together. The resulting mosaic image is shown in Fig. 3. The infalling group around LEDA 87445 is highlighted on the mosaic, together with the position of the background pointing.

\subsubsection{Spectral analysis}

We used Xselect to extract spectra in the regions of interest. We used the tools xisrmfgen and xissimarfgen to extract the appropriate response files for our regions, The data of the

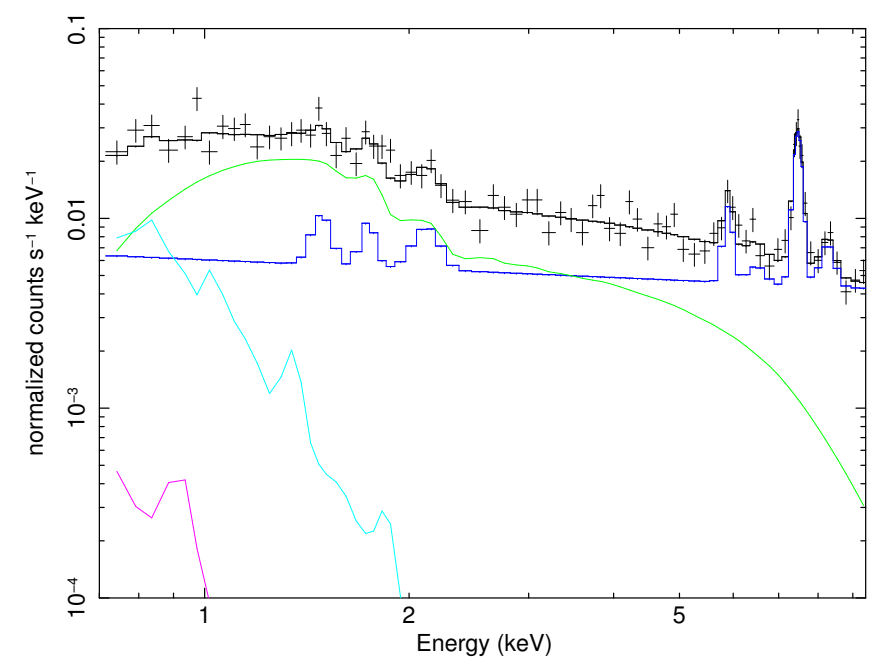

Fig. 4. Suzaku front-illuminated spectrum of the background region shown in Fig. 3 and best-fit model (black line). The dark blue curve shows the NXB model, while the sky background components are shown in green (CXB), cyan (Galactic halo), and purple (local bubble).

two front-illuminated CCDs were combined, while the XIS1 spectra were considered independently and fitted jointly. The non-X-ray background spectra were estimated from the darkEarth data using xisnxbgen. Instead of subtracting the NXB from the data, we fitted the NXB spectra using a phenomenological model and added this model as an additive component for the fitting procedure; this similar to the explanation in Sect. 2.1.3 for XMM-Newton. To the best of our knowledge this is the first time the analysis of cluster outskirts is carried out by modelling the Suzaku NXB rather than subtracting it. The sky background components were measured using the offsource pointing (see Fig. 3) with the same model as described in Sect. 2.1.3. The best-fit results for the sky components are $(1.11 \pm$ $0.13) \times 10^{-6} \operatorname{arcmin}^{-2}$ (Local Bubble) $\left(2.8_{-0.8}^{+2.9}\right) \times 10^{-7} \mathrm{arcmin}^{-2}$ (Galactic halo) with a Halo temperature of $0.33_{-0.09}^{+0.06} \mathrm{keV}$, and $(7.5 \pm 0.3) \times 10^{-7} \operatorname{arcmin}^{-2}(\mathrm{CXB})$. For the APEC models (Local Bubble and Galactic halo), normalisation $\mathrm{s}$ correspond to $10^{-14} /\left(4 \pi d_{A}^{2}(1+z)^{2}\right) \int n_{e} n_{H} d V$; for the power law (CXB), the normalisation is in units of photons $\mathrm{keV}^{-1} \mathrm{~cm}^{-2} \mathrm{~s}^{-1}$ at $1 \mathrm{keV}$.

\section{Results}

\subsection{Morphology of the stripped group}

In Fig. 5 we show a zoom of the XMM-Newton X-ray emission around the accreting group situated 17 arcmin $(1.1 \mathrm{Mpc})$ southeast from the Hydra A cluster centre, and in Fig. 6 we show a CFHT/Megacam $r$-band image of the same region with the X-ray contours overlaid. A wide variety of features are revealed. The galaxy LEDA 87445 with redshift 0.05745 (Smith et al. 2004), which is similar to the Hydra A cluster redshift, is coincident with the X-ray emission peak. East and north-east of the group emission peak, we clearly detected a long excess of diffuse emission with projected length of $\sim 12 \operatorname{arcmin}(760 \mathrm{kpc})$; this huge tail bends progressively from east towards the north-east.

A sharp surface-brightness drop can be observed $\sim 1.5$ arcmin $(100 \mathrm{kpc})$ west of the central galaxy. To confirm this statement, we extracted the surface-brightness profile from the X-ray peak of the group in the western direction in a sector with position angles between $-60^{\circ}$ and $+30^{\circ}$ (measured counter-clockwise from west) using the method described in Eckert et al. (2011) (see Fig. 7). A clear break in 


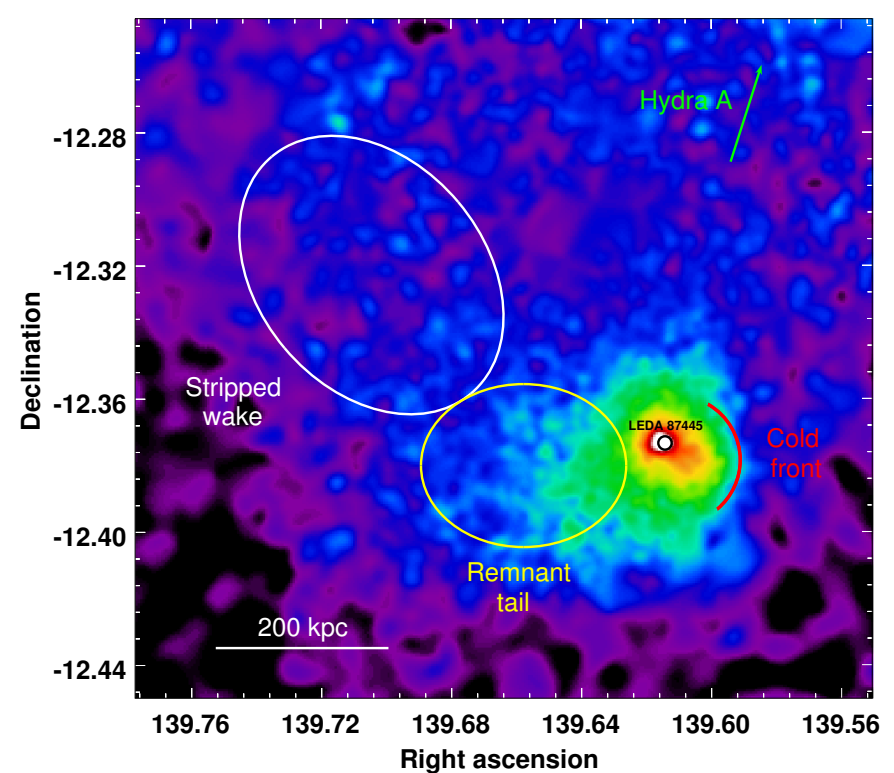

Fig. 5. Zoom of the XMM-Newton field on the LEDA 87445 group. Several noticeable features (central galaxy, cold front, remnant tail, stripped wake) are highlighted.

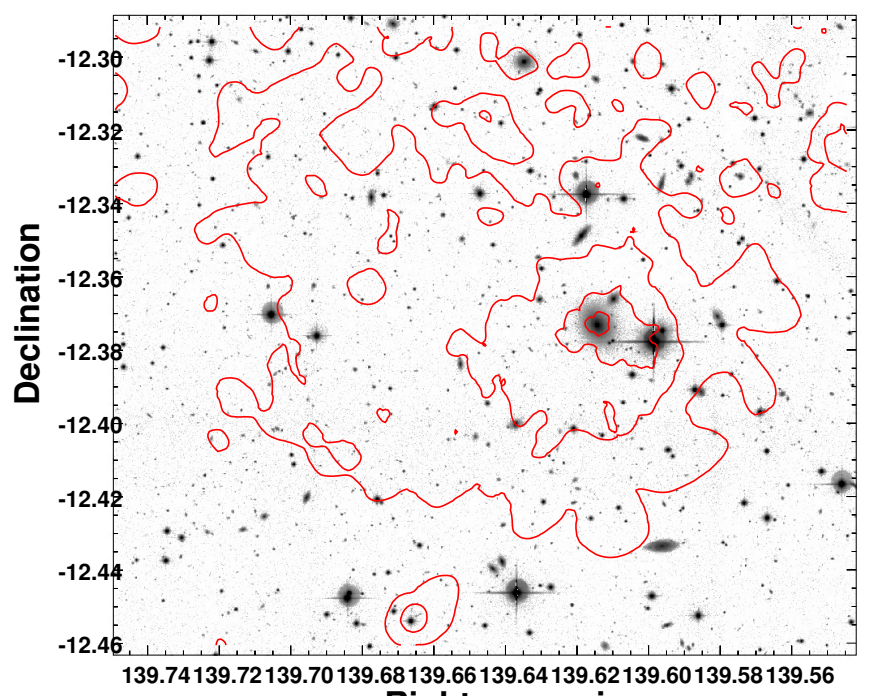

Right ascension

Fig. 6. CFHT/Megacam $r$-band image of the region around the LEDA 87445 group with the X-ray contours overlaid in red.

the surface-brightness profile can be seen 1.5 arcmin from LEDA 87445, which indicates the presence of a contact density discontinuity. We modelled the surface-brightness profile using a broken power-law density profile projected along the line of sight (Owers et al. 2009) and convolved with the XMM-Newton PSF (see Rossetti et al. 2013). This model provides a good fit to the data $\left(\chi^{2}=14.25 / 19\right.$ d.o.f. $)$ and returns a density jump $n_{\text {in }} / n_{\text {out }}=2.5_{-0.6}^{+0.8}$.

\subsection{Spectral properties}

We extracted EPIC spectra of the group and its long tail from the regions shown in Fig. 8 (left panel) and analysed them following the prescription given in Sect. 2. Spectra in similar regions were also extracted from the Suzaku data (Fig. 8, right panel). The

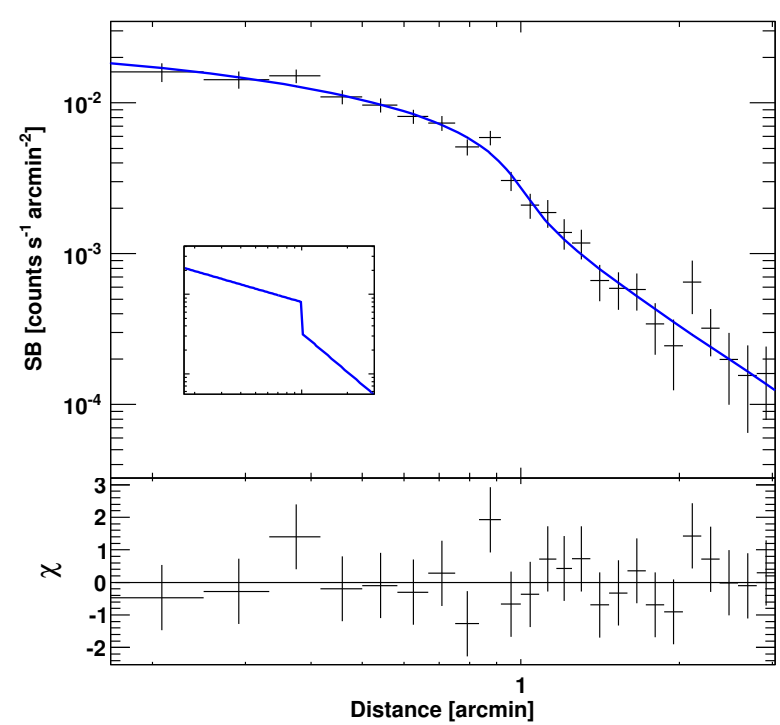

Fig. 7. EPIC surface-brightness profile from the tip of the LEDA 87445 group in a narrow westwards sector corresponding with the cold front. The solid line is the best-fitting broken power law (EPIC-PSF modelling is included). The inset shows the corresponding deprojected density model.

regions were chosen to trace the morphology of the observed feature as closely as possible.

The model fitting for each region was performed jointly on the three EPIC detectors for the XMM-Newton data and on the three XIS detectors for the Suzaku data. In Fig. 10 we only show the EPIC-pn spectra for clarity, whereas the XISO and XIS3 spectra were summed and we show the summed frontilluminated spectra in Fig. 11. The best-fit parameters for the various regions are reported in Tables 1 and 2 .

In the case of EPIC, we verified that the addition of an APEC spectral model in the source component (Sect. 2.1.3), which takes into account the residual emission of the cluster Hydra A at the distance of the group, did not significantly change any result reported. We also checked the robustness of our results against a possible cosmic variation of the CXB component in the EPIC spectra by varying the CXB normalisation by $\pm 50 \%$ in the spectral models of each region. We found that the spectral parameters remain almost unchanged and always remain within the statistical $1-2 \sigma$ uncertainties.

Before proceeding with a detailed spectral analysis of the faint emission in the tail, we decided to perform a crude, but robust, measure of this emission by comparing it to the cluster emission immediately south of the tail. From the vignettingcorrected EPIC mosaic image of Hydra A (Fig. 1), we computed the mean surface brightness (SB) in the regions of the group and tail (white regions in Fig. 8); for comparison, we also computed the mean SB in five regions nearby the group, which are shown as green circles in the same figure. The results are plotted in Fig. 9, where red circles are the SB from the tip of the group and along the tail, and squares are the SB of the nearby regions. There is clear evidence of an SB excess in the tail of the group with respect to the nearby background regions. If we consider two regions, one in the group and one in the background that are almost at the same (projected) radial distance from the core of the Hydra A, such as for instance the regions named in Fig. 8 Tail 8 and the SW, we find that the SB in the tail is higher by a factor of $\sim 2-3$ with respect to that measured in the background 

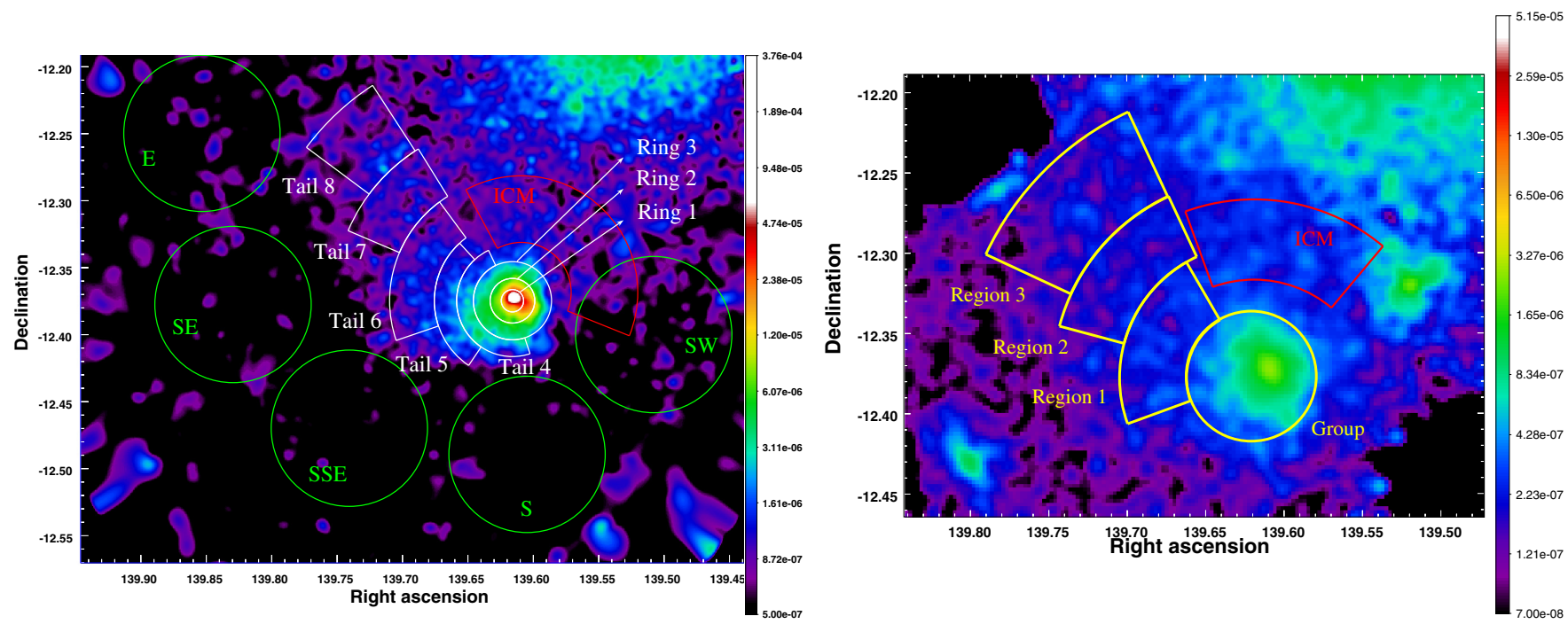

Fig. 8. Left panel: XMM-Newton image of the LEDA 87445 group with the extraction regions used for the spectral analysis. White regions are chosen to match the LEDA group and its long tail of faint emission, the red sector is used to measure the ICM temperature nearby the group, and the green circles are used to estimate the surface brightness of the ICM at various positions around LEDA. Right panel: Suzaku image of the LEDA group with, in yellow and red, the regions used for the spectral analysis of the structure and ICM, respectively. In both panels the labels of the regions are the same used in Tables 1 and 2 reporting the spectral analysis results.

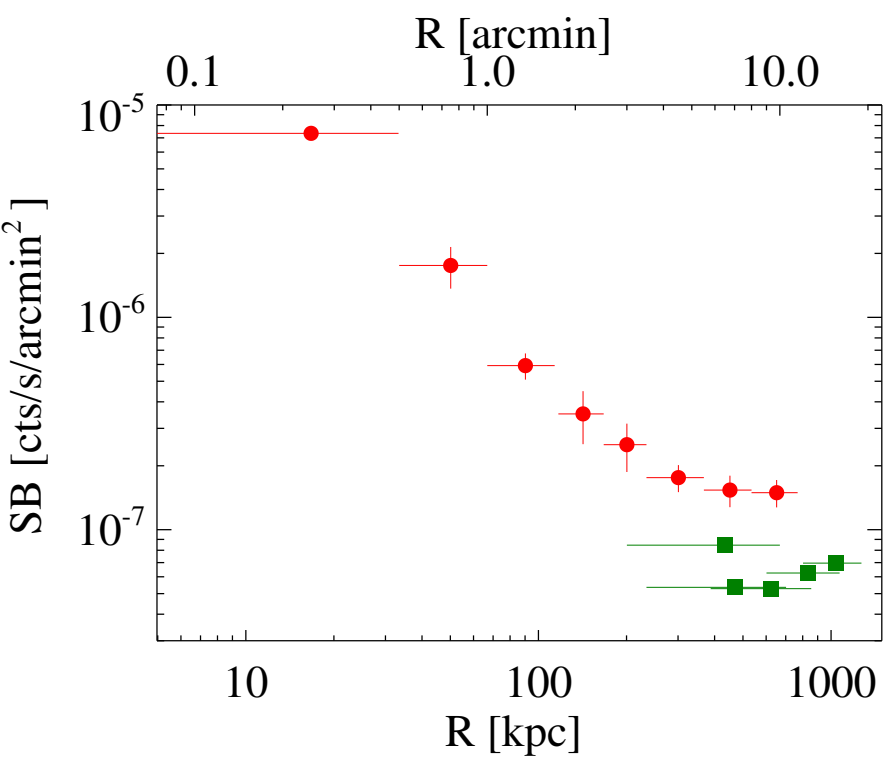

Fig. 9. EPIC surface brightness in the [0.7-1.2] keV band for the group and tail regions (red circles that correspond to the white regions in Fig. 8) and for five regions surrounding the group (green squares that correspond to the green regions in Fig. 8).

region. This SB excess in the tail can only be attribute to extra emission, since contaminations from the Galactic halo foreground or residual ICM emission affect all the considered regions at the same level.

Having established the high significance of the tail emission, we now turn to the results of the spectral analysis to better characterise it. The normalisations obtained from the analysis of the EPIC and XIS data are significantly different (see Tables 1 and 2), with those measured by XIS higher by a factor $\sim 70 \%$ than those measured by EPIC. This effect is likely due to the presence of the nearby bright Hydra A core just outside the Suzaku field of view that produces stray light and contaminates our Suzaku observation (Mori et al. 2005). To estimate the stray light effect, we considered the Suzaku region named Sector 3, where we measure a flux of $8 \times 10^{-14} \mathrm{erg} \mathrm{cm}^{-2} \mathrm{~s}^{-1}(0.5-2 \mathrm{keV}$ band). Considering that the total flux of Hydra A within 15 arcmin radius is $2.6 \times 10^{-11} \mathrm{erg} \mathrm{cm}^{-2} \mathrm{~s}^{-1}$ and that the stray light at this radius is $\sim 0.1 \%$, the contribution from the Hydra A core in Sector 3 is roughly $1 / 3$ of the flux. If we add on top of that the contribution from the LEDA 87445 group itself, which is less bright, but closer to the Sector 3 region, this very likely resolves the discrepancy between Suzaku and XMM-Newton normalisations. The stray light could also be the cause of the lower metallicity measured with Suzaku in the tail, since the extra emission would raise the observed continuum resulting in an underestimation of the metallicity.

\subsubsection{Temperature of the ICM around the LEDA group}

We estimated the ICM temperature of Hydra A close to the LEDA group with both XMM-Newton and Suzaku data. The extraction regions for these measurements are shown in red in Fig. 8; they are at $\sim 15$ arcmin from the Hydra A core for EPIC and $\sim 13$ arcmin from the core for XIS. We furthermore measured the ICM temperature from Suzaku data in a region west of the Hydra A core at the same distance from the centre as the LEDA galaxy (i.e. 17 arcmin), but far from the group emission (see red region in Fig. 3). The results are given in Tables 1 and 2. The ICM temperature at such large cluster radii is difficult to measure because of the low surface brightness and high background level in the EPIC spectrum, and because of the stray light in that of the XIS. Nevertheless, we find that the three ICM measures agree within the statistical errors and are $\sim 2-3 \mathrm{keV}$.

By comparing the normalisation per $\operatorname{arcmin}^{2}$ of the two XIS, we note that the normalisation of the SW region is lower that those measured in the region close to the LEDA group. This difference is due to both to the larger distance from the Hydra A core and to the smaller stray light contribution in the west region (where the contaminating group is far away). Moreover, the ICM normalisation measured with EPIC is higher than that of the west region measured with XIS because of the presence of a 

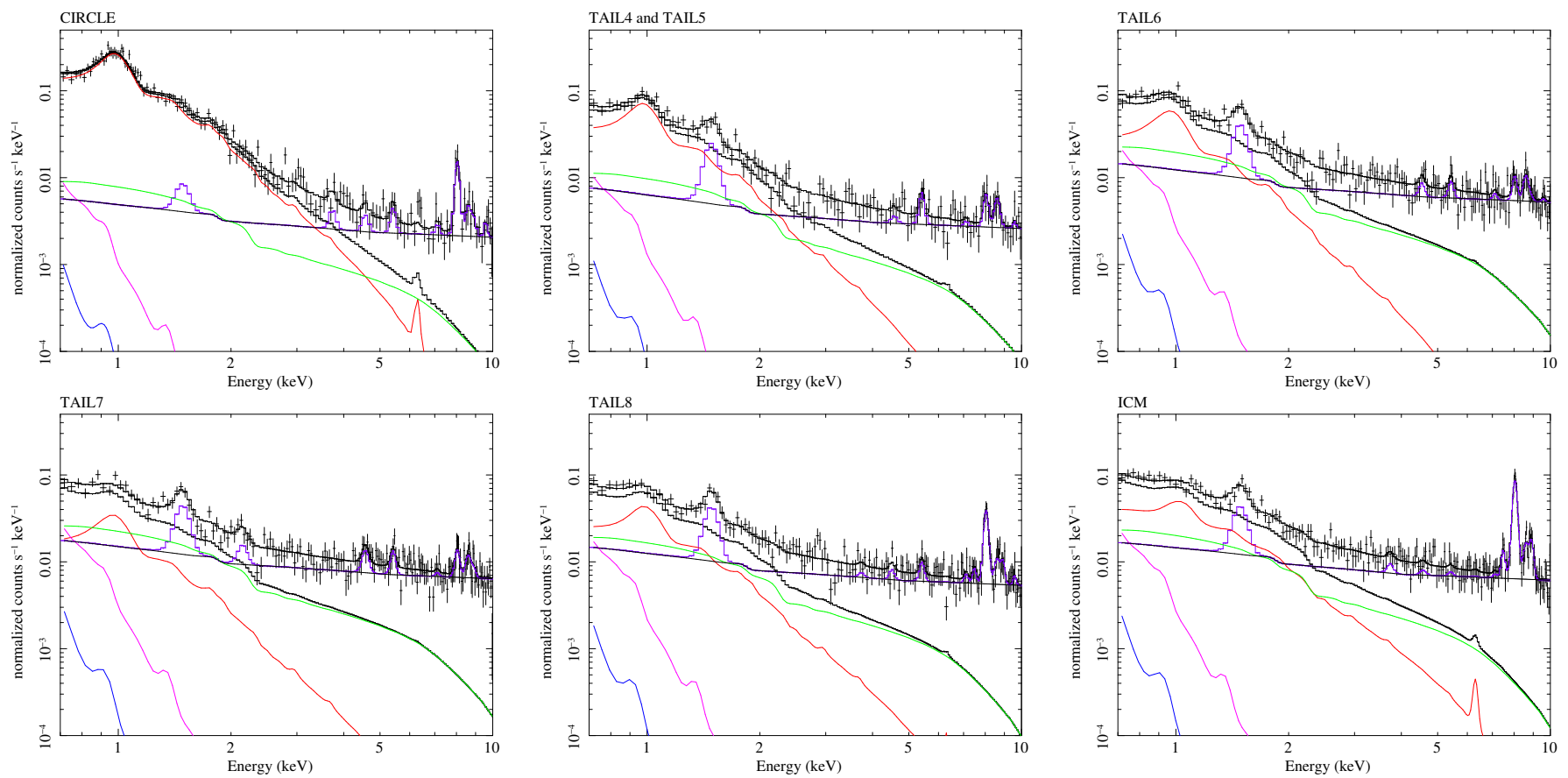

Fig. 10. EPIC/pn spectra for the regions defined in Fig. 8 (the EPIC spectra were fitted simultaneously but here we show only the pn spectra and their spectral components for clarity purposes). The solid lines show the various components used for the fitting procedure: the source (red), the NXB (blue), the CXB (green), the Galactic halo emission (magenta), and the local hot bubble (cyan). Bottom right: EPIC/pn spectrum of the local ICM in the region close to the tip of the infalling group.

Table 1. XMM: best-fit parameters for the various regions defined in Fig. 8 and for the local ICM around the tip of the group emission.

\begin{tabular}{lcccc}
\hline \hline Region & Distance [arcmin] & $k T[\mathrm{keV}]$ & \multicolumn{1}{c}{$Z_{\mathrm{Fe}}$} & $K\left[\mathrm{arcmin}^{-2}\right]$ \\
\hline Circle & $0.0-1.7$ & $1.33_{-0.01}^{+0.01}$ & $0.24_{-0.01}^{+0.02}$ & $6.4_{-0.2}^{+0.2} \times 10^{-5}$ \\
Ring 1 & $0.0-0.5$ & $1.27_{-0.01}^{+0.01}$ & $0.33_{-0.03}^{+0.03}$ & $2.4_{-0.1}^{+0.1} \times 10^{-4}$ \\
Ring 2 & $0.5-1.0$ & $1.36_{-0.06}^{+0.01}$ & $0.24_{-0.02}^{+0.02}$ & $1.0_{-0.1}^{+0.1} \times 10^{-4}$ \\
Ring 3 & $1.0-1.75$ & $1.42_{-0.05}^{+0.05}$ & $0.19_{-0.03}^{+0.03}$ & $3.6_{-0.2}^{+0.2} \times 10^{-5}$ \\
Tail 4 & $1.75-2.5$ & $1.35_{-0.03}^{+0.05}$ & $0.18_{-0.03}^{+0.03}$ & $2.2_{-0.2}^{+0.2} \times 10^{-5}$ \\
Tail 5 & $2.5-3.5$ & $1.28_{-0.05}^{+0.04}$ & $(a)$ & $7.0_{-1.1}^{+1.2} \times 10^{-6}$ \\
Tail 6 & $3.5-5.5$ & $1.27_{-0.05}^{+0.04}$ & $0.14_{-0.04}^{+0.05}$ & $7.1_{-1.1}^{+1.1} \times 10^{-6}$ \\
Tail 7 & $5.5-8.0$ & $1.28_{-0.08}^{+0.06}$ & $0.09_{-0.03}^{+0.04}$ & $6.6_{-0.9}^{+1.0} \times 10^{-6}$ \\
Tail 8 & $8.0-11.5$ & $1.25_{-0.11}^{+0.09}$ & $0.05_{-0.02}^{+0.03}$ & $1.3_{-0.2}^{+0.3} \times 10^{-5}$ \\
ICM & $2.2-5.2$ & $2.44_{-0.20}^{+0.21}$ & 0.2 & $8.8_{-0.3}^{+0.6} \times 10^{-6}$ \\
\hline
\end{tabular}

Notes. See Fig. 10 for the best fit and the various spectral components. Column description: 1: region as defined in Fig. 8. Circle is the central circular region with radius 1.7 arcmin enclosing the regions Ring 1, 2, and 3. 2: distance from the group peak in $\operatorname{arcmin}\left(1 \mathrm{kpc}\right.$ at $z_{\mathrm{LEDA}}=0.0575$ corresponds to $0.898 \mathrm{arsec}$ ). 3: best-fit temperature in $\mathrm{keV}$. 4 : metal abundance ${ }^{(a)}$ the abundance reported for region Tail 4 in the table is measured

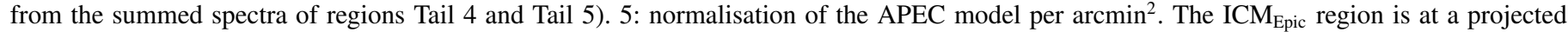
distance from the cluster centre of $\sim 15$ arcmin.

Table 2. Suzaku: best-fit parameters for the various regions defined in Fig. 8 and for the local ICM around the tip of the emission.

\begin{tabular}{lcccc}
\hline \hline Region & Distance [arcmin] & $k T[\mathrm{keV}]$ & $Z_{\mathrm{Fe}}$ & $K\left[\mathrm{arcmin}^{-2}\right]$ \\
\hline Group & $0-2.5$ & $1.26_{-0.03}^{+0.03}$ & $0.20_{-0.02}^{+0.03}$ & $1.11 \pm 0.07 \times 10^{-4}$ \\
Region 1 & $2.5-5$ & $1.23_{-0.08}^{+0.07}$ & $0.08_{-0.03}^{+0.04}$ & $2.13 \pm 0.31 \times 10^{-5}$ \\
Region 2 & $5-7.5$ & $1.24_{-0.14}^{+0.12}$ & $0.04_{-0.02}^{+0.03}$ & $2.09 \pm 0.39 \times 10^{-5}$ \\
Region 3 & $7.5-11$ & $1.49_{-0.32}^{+0.20}$ & $0.06_{-0.04}^{+0.07}$ & $1.30 \pm 0.19 \times 10^{-5}$ \\
ICM & $4.5-7.5$ & $2.68_{-0.31}^{+0.51}$ & 0.2 & $1.56 \pm 0.29 \times 10^{-5}$ \\
ICM-SW & & $3.0_{-0.9}^{+1.31}$ & 0.2 & $3.5 \pm 0.4 \times 10^{-6}$ \\
\hline
\end{tabular}

Notes. Column description: 1: region as defined in Fig. 8. 2: distance from the group tip in arcmin. 3: best-fit temperature in keV. 4: metal abundance. 5: normalisation of the APEC model per $\operatorname{arcmin}^{2}$. 6: minimum c-statistic and number of degrees of freedom. The ICM region is at the projected distance of $\sim 13$ arcmin to the cluster centre (see right panel of Fig. 8); the ICM-SW region is at the projected distance of $\sim 17.5$ arcmin south-west to the cluster centre (see red circle in Fig. 3). 

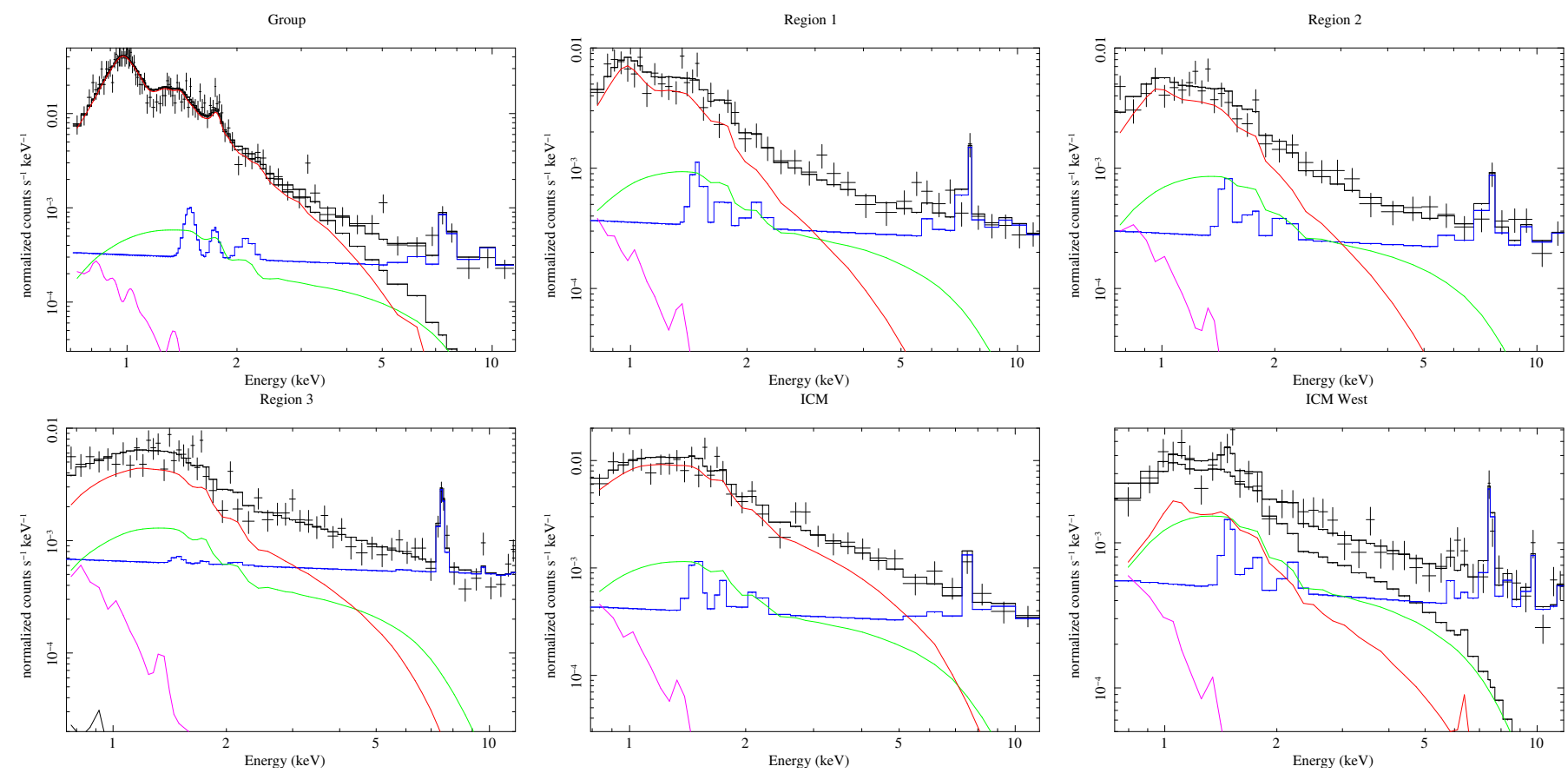

Fig. 11. Suzaku/XIS spectra of the subgroup. The fit was performed using all three XIS detectors, however for clarity purposes we only plot the spectra of the sum of the two FI units (XIS0+XIS3) and their spectral components. The solid lines show the various components used for the fitting procedure; the colour code for the different spectral components is the same as in Fig. 10. The last two panels are the spectrum of the local ICM. The panel labelled ICM refers to the region close to the tip of the infalling group and shown in Fig. 8 (right panel); the panel labelled ICM-SW is the measure in the circular region shown in red in Fig. 3.

surface-brightness excess upstream of the group motion, whose origin is discussed in Sect. 4.

\subsection{Optical data and kinematical analysis}

Optically, Hydra A is a medium-sized cluster with Abell richness class $R=0$ (Abell et al. 1989). Few redshifts are reported in the literature. We analysed the largest available homogeneous sample, that of 42 galaxies with a line-of-sight (LOS) velocity $(V=c z)$ published by Smith et al. (2004). A detailed description of our analysis is given in Appendix A; below we report only the main results.

We adopted as the centre of the Hydra A cluster the position of its brightest cluster galaxy (BCG), which has a LOS velocity of $V_{\mathrm{BCG}}=16452 \mathrm{~km} \mathrm{~s}^{-1}$, and we applied the DEDICA reconstruction method to investigate the LOS velocity distribution to the galaxies in the Smith et al. (2004) sample (e.g. Girardi et al. 1998, and references therein). In Fig. 12 we plot the resulting sample of 36 galaxies assigned to the redshift density peak of Hydra A and the LOS velocity distribution of 33 fiducial cluster members, shown in the inset of the figure. The mean cluster redshift derived from the 33 fiducial members is $\langle z\rangle=0.0548 \pm 0.0004$, i.e. $\langle V\rangle=(16421 \pm 121) \mathrm{km} \mathrm{s}^{-1}$ and the LOS velocity dispersion is $\sigma_{V}=723_{-72}^{+93} \mathrm{~km} \mathrm{~s}^{-1}$.

The bright galaxy LEDA 87445 is a cluster member lying in the high velocity part of the cluster-galaxies velocity distribution with a LOS velocity of $V_{\text {LEDA }}=17224 \mathrm{~km} \mathrm{~s}^{-1}$. We applied a Dressler \& Schectman test (DS-test; Dressler \& Shectman 1988) to probe whether the regions around the BCG and LEDA 87445 galaxies are populated by galaxies with different kinematics. This test allowed us to detect a substructure around LEDA 87445 with a c.l. larger than $99 \%$. In Fig. 13 we report the results for the DS-test based on the mean velocity (DS $\langle V\rangle)$ of the 33 cluster members. In the bubble-plot shown in Fig. 13, the larger symbols represent larger deviations of the local mean velocity from the global mean velocity and the colours indicate negative (blue) and positive (red) deviations. The plot shows a clear concentration of galaxies with positive deviation around the LEDA 87445 galaxy. The existence of correlations between positions and velocities of cluster galaxies is always a strong footprint of real substructures.

We conclude that a group of galaxies exsists around the LEDA 87445 galaxy and that this group is likely part of the Hydra A cluster halo.

\section{Discussion}

\subsection{The LEDA 87445 galaxy group}

Our analysis of the correlation between the position and velocities of the galaxies available in the literature, presented in Sect. 3.3, has revealed that a galaxy group with redshift similar to that of Hydra A exists around LEDA 87445. From the spectral analysis of the regions lying just inside and outside the sharp surface-brightness drop (Sect. 3.2), we found temperatures $k T_{\text {in }}=1.33 \pm 0.01 \mathrm{keV}$ and $k T_{\text {out }}=2.44 \pm 0.20 \mathrm{keV}$ (see Table 1 ). This allows us to conclude that this density discontinuity is a cold front produced by the motion of the galaxy group in the ambient ICM of Hydra A.

Within this picture the long diffuse emission north-east of the group peak can be naturally explained as a ram-pressure stripped tail of gas that initially belonged to the group. In Fig. 5 we can distinguish between the part of the tail directly connected with the emission peak and extending towards the east (the remnant tail), which is probably still gravitationally connected with the group peak emission, and the rest of the tail that points towards NE (the stripped wake), which is is probably formed by gas already stripped from the main group and ready to be mixed with the ICM. The common origin between the X-ray emitting gas 


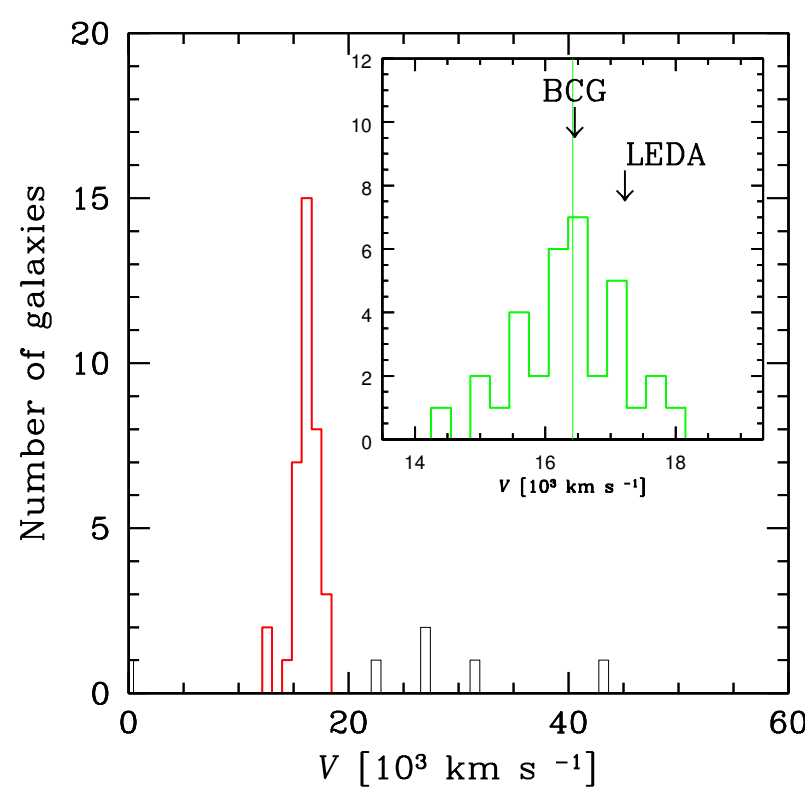

Fig. 12. Line-of-sight velocity distribution of the sample of galaxies with spectroscopic redshift. The distribution of the 36 galaxies assigned to the redshift density peak of Hydra A is highlighted in red lines. The inset figure shows the distribution of the 33 final fiducial members. The mean cluster velocity (vertical faint green line) and the velocities of Hydra A BGC and LEDA 87445 are indicated.

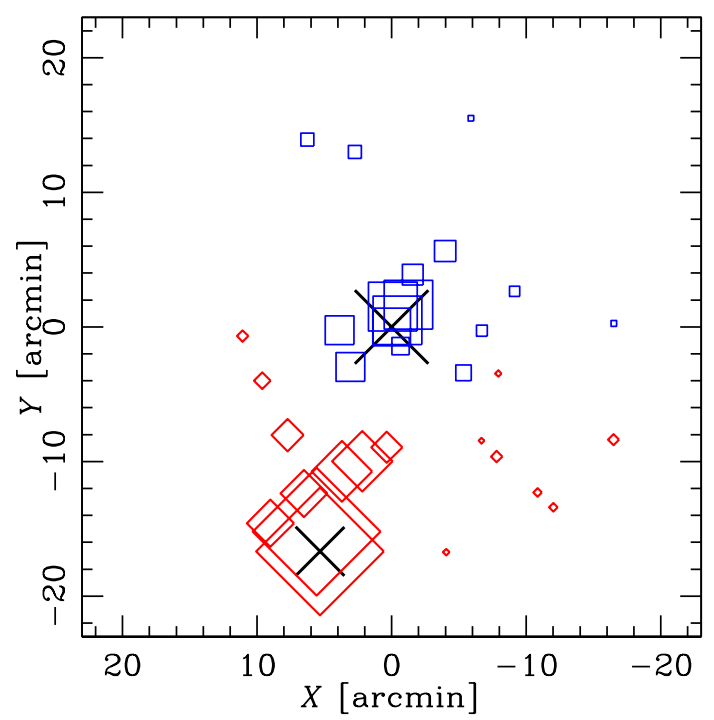

Fig. 13. DS $\langle V\rangle$ bubble plot for the 33 cluster members. The larger the symbol, the larger the deviation of the local mean velocity from the global mean velocity. Blue squares indicate negative deviations and red rotate squares positive deviations. Crosses indicate the BGC (centre) and LEDA 87445.

in the tip and all along the tail is strengthened by the thermal properties of the gas in the whole region; the constant temperature (Fig. 14, left panel) up to $\sim 760 \mathrm{kpc}$ from the peak and its lower value with respect to the ICM temperature (Tables 1 and 2) indicates the same origin of the gas.

The core of the LEDA 87445 group appears well defined, indicating that we are observing the system likely during its first core passage with a substantial part of the gas still gravitationally retained by the group.

\subsection{Thermodynamical properties of the LEDA group}

Temperature and metal abundance distributions in the group LEDA 87445 and its tail are plotted in Fig. 14, where red circles are EPIC and blue squares are XIS measurements. Both profiles are centred on the X-ray emission peak of the structure $\left(\mathrm{RA}=9^{\mathrm{h}}: 18^{\mathrm{m}}: 27.783^{\mathrm{s}}\right.$, Dec $\left.=-12^{\mathrm{o}}: 22^{\prime}: 30.66^{\prime \prime}\right)$ and follow the faint tail as shown in Fig. 8. From the mean temperature of the group (1.3 keV) and the scaling relation of Arnaud et al. (2005), we estimated the $\mathrm{R} 500$ radius of the group, $\mathrm{R} 500=497 \mathrm{kpc}$, and we used this value to rescale the profiles. Measurements by EPIC and XIS agree rather well in both profiles. The slightly lower iron abundance found by XIS beyond $200 \mathrm{kpc}$ is likely related to the stray-light and PSF effects of Suzaku, already discussed in Sect. 3.2.

We find that the temperature along the group is roughly constant at $\sim 1.3 \mathrm{keV}$, which is a value typical of the virialised halo of galaxy groups with mass of a few $10^{13} M_{\odot}$. At the centre $k T$ is $1.27 \mathrm{keV}$, which increases up to $1.42 \mathrm{keV}$ at about $100 \mathrm{kpc}$, and then decreases to 1.3 at $200-220 \mathrm{kpc}$, which is the end of the remnant tail. Beyond this point, the temperature remains constant along all the stripped wake up to $>500 \mathrm{kpc}$.

The metal abundance profile (Fig. 14, right panel) shows the typical trend observed in the core of relaxed groups and clusters, namely a higher abundance $\left(0.33 \mathrm{Z}_{\mathrm{Fe}}\right)$ at the emission peak, which then decreases moving away from the peak (e.g. Leccardi \& Molendi 2008a; De Grandi et al. 2004). In the remnant tail, between $\sim 100-200 \mathrm{kpc}$, the abundance reaches the typical value for the outermost regions of group (see review by Sun 2012 , i.e. $\sim 0.2 \mathrm{Z}_{\mathrm{Fe}}$ ), then declines even more along all the faint and patchy stripped wake to very low values. The rather low abundance in the wake could be explained, as in the case of A2142 (Eckert et al. 2014), by a multi-temperature structure of the gas; in fact, when a multiphase gas with temperatures below $\sim 2 \mathrm{keV}$ is fit with a single temperature spectral model it returns an iron abundance that is biased towards low values. This is a well studied effect known as the Fe bias (Buote 2000a,b).

The surface-brightness fluctuations observed in the stripped gas of the faint and patchy tail (see Fig. 5), likely related to gas clumpiness or to heated ICM gas mixed to the stripped gas, actually suggest that the gas is multiphase.

In Fig. 15 we show the pseudo-entropy profile of the gas, defined as $\Sigma=k T / K^{1 / 3}$, where both $k T$ and $K$ are taken from Table 1, along the tail of the group. The profile is characterised by a rapid increase as we move away from the core, which is typical of cool core systems; this is followed by an initial flat region between $60 \mathrm{kpc}$ and $160 \mathrm{kpc}$, a jump around $160 \mathrm{kpc}$, and a more extended flat region going from 160 to $500 \mathrm{kpc}$. The jump occurs roughly where the tail starts to become clumpy and likely marks the transition between the remnant tail and stripped tail. The shape of the profile suggests that the gas evolves adiabatically both in the region where it is gravitationally bound to the group and further out. However the transition between the two regimes is not adiabatic, and there is a sudden and significant increase in entropy. We note that since the gas appears to be more clumpy in the stripped than in the remnant tail, the density in the latter is likely more significantly overestimated than in the former, implying that the jump in entropy is even more pronounced than we estimate. While the exact nature of the process responsible for heating the gas is unclear, its abrupt nature suggests that it is related to the stripping process itself. 

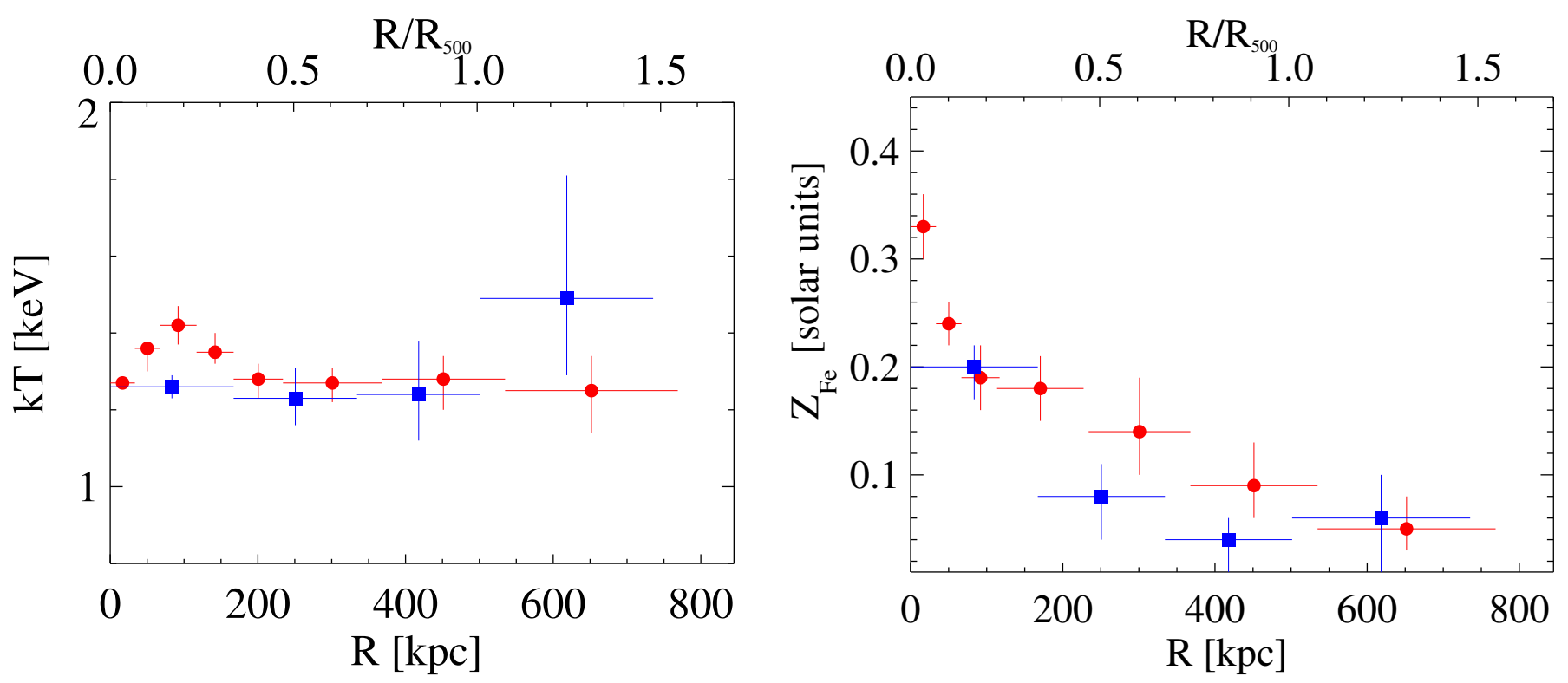

Fig. 14. Left panel: temperature profile of the LEDA 87445 group and tail. Right panel: metal abundance profile. In both panels filled red circles are EPIC data and filled blue squares are XIS data. The radius is the distance from the group emission peak of the regions shown in Fig. 8.

\subsection{ICM emission north-east and north-west of the group}

As shown in Figs. 5 and 8, the ICM emission north-west and north-east of the LEDA group is more intense than in other cluster regions located at the same radial distance from the centre. If, as the position of the cold front suggests, the group is currently moving west, the upstream enhancement is likely due to compression by the group itself. Moreover if, as suggested by estimates provided in Sect. 4.5, the motion is subsonic, the compression is adiabatic and the entropy we measure in this region should be similar to that of neighbouring regions, where the lower surface brightness prevents direct measures. Conversely, the enhancement north-east of the group is likely related to tidal effects. Indeed, as the group moves through the atmosphere of the cluster, gas is displaced towards it (see Fig. 3, panels 3 to 5, in Ascasibar \& Markevitch 2006). It is worth noticing that the contiguity between the gas in the tail (see Sect. 4.2) and that in the north-east excess makes it difficult to provide a clear separation between the two.

\subsection{Ram-pressure stripping properties}

The LEDA 87445 group has a peak and a rather long tail $(760 \mathrm{kpc})$. We performed a simple estimate of the velocity of the moving group by assuming that the gas at the tip of the structure is in pressure equilibrium with its surrounding ICM (Markevitch et al. 2000),

$P_{\mathrm{ICM}}+\rho v_{\text {rel }}^{2}=P_{\text {group }}$,

where $v_{\text {rel }}$ is the velocity with which the group is moving through the cluster, $\rho$ is the density of the ambient ICM, and $P_{\text {ICM }}$ and $P_{\text {group }}$ are the pressure associated with the ambient ICM and the tip of the group, respectively. We began by estimating gas densities on the two sides of the cold front from the surfacebrightness profile (see Fig. 7). From the spectral analysis reported in Sect. 3.2, we measured temperatures of $1.3 \mathrm{keV}$ for the for the downstream region and $2.44 \mathrm{keV}$ for the ICM region. By combining density and temperature measures, we derived estimates for $P_{\mathrm{ICM}}$ and $P_{\text {group }}$. Finally by applying Eq. (1) we obtained an estimated velocity of $440 \pm 170 \mathrm{~km} / \mathrm{s}$, which is about half the speed of sound, $c_{\mathrm{s}}=\sqrt{\frac{\gamma k T}{\mu m_{\mathrm{p}}}}=820 \pm 60 \mathrm{~km} \mathrm{~s}^{-1}$, for a plasma of $2.44 \mathrm{keV}$.

The detection of a cold front west of the group centre allowed us to estimate the velocity of the moving group through the stagnation point argument developed by Vikhlinin et al. (2001). This estimate should return a more precise estimate of the velocity, since it is using the measurement of the density jump directly at the front. From the measurement of the density jump at the front $\left(n_{\text {in }} / n_{\text {out }}=2.5_{-0.6}^{+0.8}\right.$, see Sect. 3.1) and the temperature difference between the regions inside and outside the front (see Table 1), we estimate a pressure jump $p_{\text {in }} / p_{\text {out }}=1.4_{-0.3}^{+0.5}$, which through the stagnation point argument corresponds to a Mach number $\mathcal{M}=0.6_{-0.4}^{+0.5}$ (see Vikhlinin et al. 2001; Markevitch \& Vikhlinin 2007). Given the sound speed in the medium beyond the cold front, we estimate that the group is moving at a speed of $510_{-340}^{+430} \mathrm{~km} \mathrm{~s}^{-1}$ relative to the main cluster.

Thus, from both the estimates of the velocity, the group is falling onto the main cluster at, roughly, half the sound speed. Under the assumption that the group has been moving at a constant velocity, we find that the gas in the outermost region of the structure was expelled some $1.4-1.6$ Gyr ago.

Assuming that the extension of the emission on the line of sight is the same as in the plane of the sky, we derived a crude estimate of the gas mass that resides in the tail from the normalisations of Tail regions 4 to 8 (see Table 1 ), finding $1.4 \times 10^{11} M_{\odot}$. A similar analysis for the Ring regions 1 to 3 leads to a mass of $1.1 \times 10^{11} M_{\odot}$ for the tip of the structure. Thus our analysis seems to indicate that roughly $60 \%$ of the observed gas mass is located in the tail. From the temperature of the substructure, we estimate a total mass of the order of a few $(2-5) \times 10^{13} M_{\odot}$ (Sun et al. 2009). Assuming a gas fraction of 5\% (Sun et al. 2009) a group like ours should have a gas mass of the order of $1.5 \times 10^{12} M_{\odot}$, which is roughly a factor of 6 larger than what we estimate to be currently associated with either the group or its tail. Assuming that all the excess emission observed in the Hydra A SE region is associated with gas once residing in the group's potential well, we arrive at a conservative upper limit for the total gas mass of the group of $5 \times 10^{11} M_{\odot}$, which is still low by a factor of 2.5 with respect to expectations. While gas mass estimates for groups are 


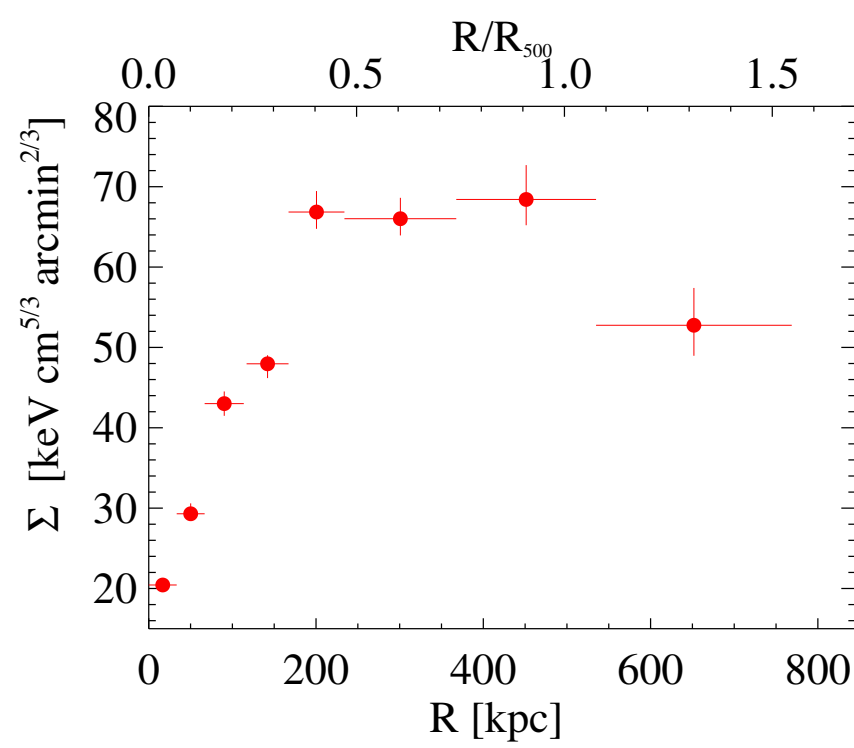

Fig. 15. Pseudo-entropy profile of the LEDA 87445 group and tail regions (EPIC data only).

clearly less precise than those for clusters, these results seem to suggest that our group may have already lost a significant fraction of gas prior to the stripping event we are currently observing. Irrespective of what the starting gas mass of our group might have been, the current data is already sufficient to inform us that a significant fraction of the gas has been stripped from the group. Assuming that the part of the tail nearest the tip be made of gas that is still gravitationally bound to the group, we estimate the stripped gas to be between 1 and $5 \times 10^{11} M_{\odot}$, i.e. one-half and four-fifths of the total gas mass, depending on whether the excess gas in the Hydra A SE region was donated by the group.

As in the case of the group falling onto A2142 (Eckert et al. 2014), we performed an estimate of the timescale required for the gas in the tail to be heated up to the temperature of the ambient ICM. We assumed the length scale over which the temperature gradient develops to be $100 \mathrm{kpc}$. We also adopted an ICM temperature of $2.44 \mathrm{keV}$ and an electron density for the ICM surrounding the tail of $7 \times 10^{-5} \mathrm{~cm}^{-3}$, which is estimated from the ROSAT surface-brightness profile of Hydra A, provided by Eckert et al. (2012), by excluding the sector contaminated by the group emission. Finally, adopting Eqs. (2) and (3) of Eckert et al. (2014; originally derived in Gaspari \& Churazov 2013), we found a conduction timescale of $21.1 f^{-1} \mathrm{Myr}$, where $f$ is the effective isotropic conduction suppression factor. By equating this timescale with the age of the structure, which was previously determined to be between 1.4 and $1.6 \mathrm{Gyr}$, we estimate a suppression factor of more than 70. Thus, as for the case of A2142, we find evidence for significantly suppressed conductivity.

We note that the lack of significant heating of the gas in the tail is in agreement with the flat entropy profile we measure along the stripped tail (Fig. 15). Indeed, if heating were taking place, we would expect an increase in entropy as we move away from the group. The high suppression of conduction is consistent with the survival of cold fronts and filamentary structures (Ettori \& Fabian 2000; Forman et al. 2007; Sanders \& Fabian 2013). Through the power spectrum of density perturbations, Gaspari \& Churazov (2013), Gaspari et al. (2014) also retrieved a high suppression factor in the Coma cluster. Another recent example showing the suppression of transport processes is given by Ichinohe et al. (2015) in their study of the complex structure of cluster Abell 85. The presence of quasi-linear, high-density

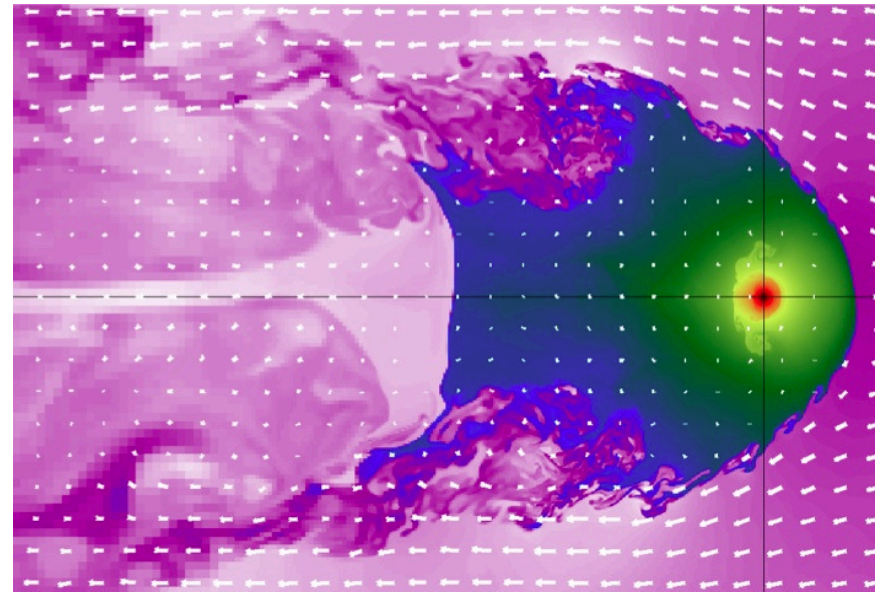

Fig. 16. From Roediger et al. (2015a). Snapshot of a high-resolution simulation of the hot atmosphere of an elliptical galaxy falling into a galaxy cluster. The bottom of the potential well is indicated with the black cross. The arrows show the velocity field of the fluid. Several features can be seen: the cold front at the peak with the KH rolls on the side, the remnant tail in the blue low-velocity area, and the stripped tail trailing behind.

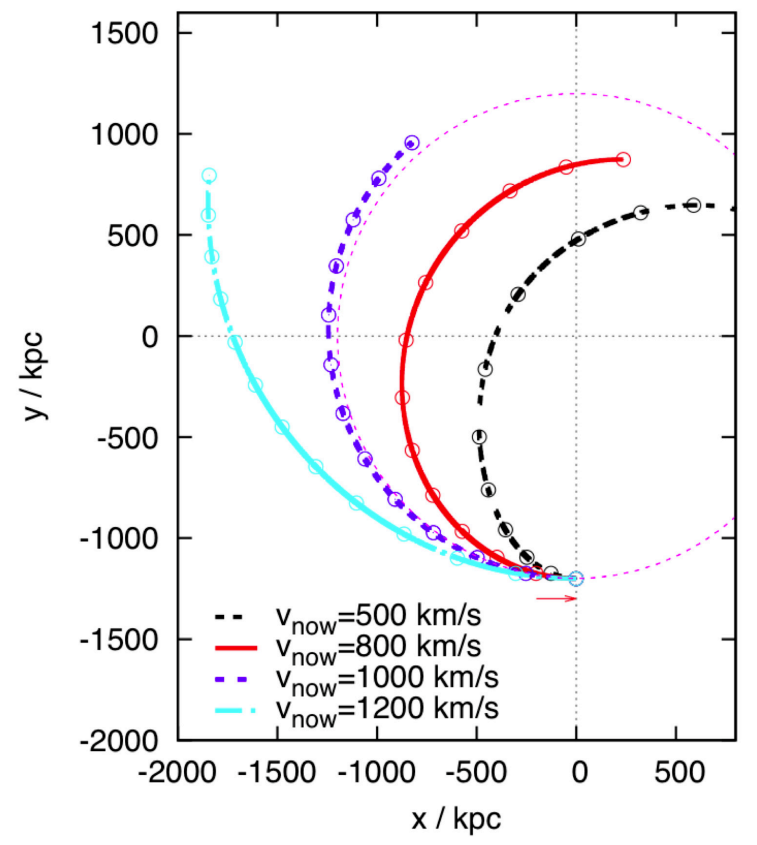

Fig. 17. Orbits of a test particle in the potential well of Hydra A moving with different velocities. The red arrow show the position of the observed LEDA 87445 group at $1.1 \mathrm{Mpc}$ from the centre of Hydra A (at coordinates 0,0 ). The dashed red circle is the Keplerian orbit (i.e. smaller orbits are gravitationally bound with the main cluster). The circles along the orbits are spaced in $0.25 \mathrm{Gyr}$ intervals.

filamentary structures spanning several kpc observed in the cores of nearby clusters, such as the Coma cluster (Sanders et al. 2013), consisting of low-entropy material that was probably stripped from merging subclusters, shows that the conduction is heavily suppressed in cluster centres as well. Most recent observations seem thus to agree on the low thermal conductivity of the ICM. Magnetic fields are presumably the most effective agents to maintain these features separately from the ICM. 


\subsection{Infalling group motion}

In the previous section we assumed that the motion of the accreting group is on the plane of the sky. This is suggested in $\mathrm{X}$-rays by the position of the cold front pointing towards the west, whereas the main Hydra A cluster is located in the northern direction, and by the shapes of tail and wake, which gradually curve from the east to the north (see Fig. 5). We postulated that the group was originally coming from the north-eastern direction with a large impact parameter and that its trajectory is being bent by the gravitational pull of Hydra A.

The stagnation point analysis leads to a velocity of the group of the order of half of the sound speed; this is the 3D velocity in the hypothesis that the orbit is not inclined too much out of the plane of the sky. However, the LOS velocity difference between the LEDA 87445 galaxy and the BCG of Hydra A, $772 \mathrm{~km} \mathrm{~s}^{-1}$ is of the same order as the cold front velocity, implying that the group is likely moving with a certain angle along the LOS, i.e $\sim 30^{\circ}-50^{\circ}$. The visibility of the cold front would be preserved with such an orbital angle. Roediger et al. (2015b) show mock $\mathrm{X}$-ray images of inclined galaxies falling into a group/cluster ICM (see their Figs. 7 and 12) in which the cold front is visible at least up to inclinations of 60 degree out of the plane of the sky. If the cold front is indeed inclined by $\sim 45^{\circ}$ with respect to the plane of the sky, then projection effects likely lead to an underestimation of the group velocity from the stagnation point analysis.

To fully understand the motion of the group, more detailed $\mathrm{X}$-ray and optical observations, as well as detailed analyses of mock observations, would be highly useful.

\subsection{Comparison with numerical simulations}

Ram-pressure-stripped, X-ray-emitting wakes and tails are observed trailing galaxies infalling onto groups and clusters. Several recent works have addressed the subject with numerical simulations (e.g. Cen et al. 2014; Vijayaraghavan \& Ricker 2015; Roediger et al. 2015a,b, and references therein).

In Fig. 16 we show an example of a simulated elliptical galaxy infalling radially in the ICM ambient from Roediger et al. (2015a). A sharp cold front lies at the tip of the structure with KH rolls on its side. The wake of the infalling structure is composed of a dense region of bound gas that has been pushed back by the ram pressure (dubbed the remnant tail in Roediger et al. 2015a) and of the actual wake of thin gas stripped through $\mathrm{KH}$ instabilities (the stripped wake). The similarity between the observed morphology of our group (Fig. 5) and the simulated system in Fig. 16 is striking. In addition to the cold front, we also observe the remnant tail of the moving group and the faint, patchy stripped wake.

Vijayaraghavan \& Ricker (2015), simulating the ram pressure effects on a population of galaxies in isolated cluster environments, found that cluster galaxies are stripped of their gas by the ICM, and that the stripped gas trails the galaxy orbits in the form of wakes before mixing with the ICM. Under the hypothesis that the morphology of the infalling LEDA 87445 group, with its bent tail of stripped gas and westwards cold front, also marks the recent part of the group orbit, we derived a rough estimate of the orbit as follows. We modelled the Hydra A cluster as a spherical gravitational potential filled with a hydrostatic ICM. Thus, we could calculate the radial gravitational acceleration in the cluster as a function of radius from the observed ICM density (Eckert et al. 2012) and temperature profiles (Simionescu et al. 2009). We then placed a test particle, i.e a particle that follows only the gravity of the cluster potential, at the current position of LEDA 87445 , i.e. $1.1 \mathrm{Mpc}$ south of the cluster centre. Initializing the test particle with the negative of the current velocity of LEDA $87445, v_{\text {now }}$, we could trace back the particles motion in the cluster potential in time. Figure 17 shows the resulting orbits assuming a purely tangential velocity of the group for several magnitudes of $v_{\text {now }}$ (the circles along the orbit in the Figure are spaced in 0.25 Gyr intervals). This estimate neglects any deceleration of the group by dynamical friction.

For this cluster model, a current velocity of the test particle such as that we inferred for the LEDA 87445 group from the stagnation point analysis, $510_{-340}^{+430} \mathrm{~km} \mathrm{~s}^{-1}$, would suggest that the group moved on a bound orbit. However if, as discussed in Sect. 4.5, our estimate of the velocity is biased low because of inclination effects, then the group is moving on an almost Keplerian orbit and likely making its first passage.

\section{Conclusions}

In this paper we reported the discovery of the ram-pressure stripped infalling galaxy group in the outskirts of the poor cluster Hydra A/A780. We used data from XMM-Newton and Suzaku observations, as well as optical data drawn from the literature, to study the properties of this accreting subgroup. We summarise our main results as follows:

1. The X-ray emission peak of the group is at $1.1 \mathrm{Mpc}$ southeast of the Hydra A cluster centre. Its X-ray morphology is peculiar as it shows a tail and wake that is $760 \mathrm{kpc}$ long (in projection), and, in the opposite direction, a cold front. These features are likely produced by the motion of the infalling group within the ICM.

2. The optical kinematical analysis reveals that the X-ray emission peak coincides with the early-type galaxy LEDA 87445 and that around this galaxy there is an overdensity of galaxies, at a c.l. larger that $99 \%$.

3. The X-ray morphology and optical data, also compared with recent simulations of galaxies infalling in the ICM, give hints about the orbit of the LEDA group that lead us to conclude that the group is likely at its first passage onto Hydra A.

4. The gas temperature of the LEDA 87445 group emission peak and along its tail and wake is roughly constant $(\sim 1.2-1.3 \mathrm{keV})$ and is lower than the ambient ICM $(\sim 2.4-3 . \mathrm{keV})$. This temperature is typical of the virialised plasma of a galaxy group with a mass of a few $10^{13} M_{\odot}$. We estimate that the stripped gas is between 1 and $5 \times 10^{11} M_{\odot}$, i.e. somewhere between one-half and four-fifths of the total observed gas mass.

5. The velocity of the moving group, estimated through the cold front stagnation point argument, is $510_{-430}^{+340} \mathrm{~km} \mathrm{~s}^{-1}$ relative to the main cluster (corresponding to a Mach number of $0.6_{-0.4}^{+0.5}$ ) and is comparable to half the sound speed. We find that the gas in the outermost region of the structure was expelled 1.4-1.6 Gyr ago.

6. We estimated the timescale required for the gas in the tail to be heated up to the temperature of the ambient ICM, and compared this timescale with the age of the structure (i.e. 1.4-1.6 Gyr). We found that the suppression factor is at least two orders of magnitude suppressed compared with Spitzer conductivity, which is in line with the findings for other clusters (e.g. Eckert et al. 2014; Ichinohe et al. 2015). These long survival times of the stripped gas within the ambient ICM would have important implications on the evolution of the hot gas content in galaxy clusters, as the cold gas 
in the stripped structures could eventually reach the core of the main cluster.

7. Using the relative radial velocity between the galaxy LEDA 87445 and the Hydra A BCG, we find that the orbit of the accreting LEDA 87445 group could be inclined with an angle of $\sim 45^{\circ}$ with respect to the plane of the sky; therefore, the velocity of the group estimated from the stagnation point analysis would be underestimated as a result of projection effects. However, more detailed hydrodynamic modelling of this system is required to resolve the full three-dimensional motion of this system.

The striking similarity between the infalling group discovered here and hydrodynamical simulations of similar structures (see Figs. 5 and 16) makes this system an ideal target to test our understanding of ram-pressure stripping and ICM physics, for example by comparing the morphology of the tail and searching for features like $\mathrm{KH}$ instabilities. Deeper, higher resolution observations however are required to perform such a detailed comparison.

Acknowledgements. We thank Henk Hoekstra and Remco van der Burg for kindly providing us the stacked CFHT image. We acknowledge financial contribution from contract PRIN INAF 2012 (a unique dataset to address the mos compelling open questions about X-ray galaxy clusters). M.G. is supported by NASA through Einstein Postdoctoral Fellowship Award Number PF-160137 issued by the Chandra X-ray Observatory Center, which is operated by the SAO for and on behalf of NASA under contract NAS8-03060. This research is based on observations obtained with XMM-Newton, an ESA science mission with instruments and contributions directly funded by ESA Member States and the USA (NASA), and has made use of data obtained from the Suzaku satellite, a collaborative mission between the space agencies of Japan (JAXA) and the USA (NASA).

\section{References}

Abell, G. O., Corwin, H. G., \& Olowin, R. P. 1989, ApJS, 70, 1 Abramson, A., Kenney, J. D. P., Crowl, H. H., et al. 2011, AJ, 141, 164 Anders, E., \& Grevesse, N. 1989, Geochim. Cosmochim. Acta, 53, 197 Arnaud, M., Pointecouteau, E., \& Pratt, G. W. 2005, A\&A, 441, 893 Ascasibar, Y., \& Markevitch, M. 2006, ApJ, 650, 102

Barrena, R., Girardi, M., Boschin, W., et al. 2011, A\&A, 529, A128

Beers, T. C., Flynn, K., \& Gebhardt, K. 1990, AJ, 100, 32

Berrier, J. C., Stewart, K. R., Bullock, J. S., et al. 2009, ApJ, 690, 1292

Bird, C. M. 1994, AJ, 107, 1637

Boselli, A., Cortese, L., Boquien, M., et al. 2014, A\&A, 564, A67

Buote, D. A. 2000a, ApJ, 539, 172

Buote, D. A. 2000b, MNRAS, 311, 176

Cash, W. 1979, ApJ, 228, 939

Cen, R., Pop, A. R., \& Bahcall, N. A. 2014, PNAS, 111, 7914

Chung, A., van Gorkom, J. H., Kenney, J. D. P., \& Vollmer, B. 2007, ApJ, 659, L115

Danese, L., de Zotti, G., \& di Tullio, G. 1980, A\&A, 82, 322

David, L. P., Nulsen, P. E. J., McNamara, B. R., et al. 2001, ApJ, 557, 546

De Grandi, S., Ettori, S., Longhetti, M., \& Molendi, S. 2004, A\&A, 419, 7

De Luca, A., \& Molendi, S. 2004, A\&A, 419, 837

De Lucia, G., Weinmann, S., Poggianti, B. M., Aragón-Salamanca, A., \& Zaritsky, D. 2012, MNRAS, 423, 1277

Dolag, K., Borgani, S., Murante, G., \& Springel, V. 2009, MNRAS, 399, 497

Dressler, A., \& Shectman, S. A. 1988, AJ, 95, 985

Durret, F., Lima Neto, G. B., \& Forman, W. 2005, A\&A, 432, 809
Durret, F., Slezak, E., \& Adami, C. 2009, A\&A, 506, 637 Eckert, D., Molendi, S., \& Paltani, S. 2011, A\&A, 526, A79

Eckert, D., Vazza, F., Ettori, S., et al. 2012, A\&A, 541, A57

Eckert, D., Molendi, S., Owers, M., et al. 2014, A\&A, 570, A119

Ettori, S., \& Fabian, A. C. 2000, MNRAS, 317, L57

Fadda, D., Girardi, M., Giuricin, G., Mardirossian, F., \& Mezzetti, M. 1996, ApJ, 473,670

Forman, W., Jones, C., Churazov, E., et al. 2007, ApJ, 665, 1057

Gaspari, M., \& Churazov, E. 2013, A\&A, 559, A78

Gaspari, M., Churazov, E., Nagai, D., Lau, E. T., \& Zhuravleva, I. 2014, A\&A, 569, A67

Genel, S., Bouché, N., Naab, T., Sternberg, A., \& Genzel, R. 2010, ApJ, 719, 229

Girardi, M., Fadda, D., Giuricin, G., et al. 1996, ApJ, 457, 61

Girardi, M., Giuricin, G., Mardirossian, F., Mezzetti, M., \& Boschin, W. 1998, ApJ, 505, 74

Gunn, J. E., \& Gott, III, J. R. 1972, ApJ, 176, 1

Ichinohe, Y., Werner, N., Simionescu, A., et al. 2015, MNRAS, 448, 2971

Kalberla, P. M. W., Burton, W. B., Hartmann, D., et al. 2005, A\&A, 440, 775

Kawata, D., \& Mulchaey, J. S. 2008, ApJ, 672, L103

Kempner, J. C., Sarazin, C. L., \& Ricker, P. M. 2002, ApJ, 579, 236

Leccardi, A., \& Molendi, S. 2008a, A\&A, 487, 461

Leccardi, A., \& Molendi, S. 2008b, A\&A, 486, 359

Lubin, L. M., Brunner, R., Metzger, M. R., Postman, M., \& Oke, J. B. 2000, ApJ, 531, L5

Machacek, M., Jones, C., Forman, W. R., \& Nulsen, P. 2006, ApJ, 644, 155

Markevitch, M., \& Vikhlinin, A. 2007, Phys. Rep., 443,

Markevitch, M., Ponman, T. J., Nulsen, P. E. J., et al. 2000, ApJ, 541, 542

McCarthy, I. G., Frenk, C. S., Font, A. S., et al. 2008, MNRAS, 383, 593

McNamara, B. R., Wise, M., Nulsen, P. E. J., et al. 2000, ApJ, 534, L135

Mori, H., Iizuka, R., Shibata, R., et al. 2005, PASJ, 57, 245

Munari, E., Biviano, A., Borgani, S., Murante, G., \& Fabjan, D. 2013, MNRAS, 430, 2638

Navarro, J. F., Frenk, C. S., \& White, S. D. M. 1996, ApJ, 462, 563

Neumann, D. M., Lumb, D. H., Pratt, G. W., \& Briel, U. G. 2003, A\&A, 400, 811

Nousek, J. A., \& Shue, D. R. 1989, ApJ, 342, 1207

Owers, M. S., Nulsen, P. E. J., Couch, W. J., \& Markevitch, M. 2009, ApJ, 704, 1349

Pinkney, J., Roettiger, K., Burns, J. O., \& Bird, C. M. 1996, ApJS, 104, 1

Pisani, A. 1993, MNRAS, 265, 706

Pisani, A. 1996, MNRAS, 278, 697

Randall, S., Nulsen, P., Forman, W. R., et al. 2008, ApJ, 688, 208

Roediger, E., Kraft, R. P., Nulsen, P. E. J., et al. 2015a, ApJ, 806, 103

Roediger, E., Kraft, R. P., Nulsen, P. E. J., et al. 2015b, ApJ, 806, 104

Rossetti, M., Eckert, D., De Grandi, S., et al. 2013, A\&A, 556, A44

Sanders, J. S., \& Fabian, A. C. 2013, MNRAS, 429, 2727

Sanders, J. S., Fabian, A. C., Churazov, E., et al. 2013, Science, 341, 1365

Sato, T., Sasaki, T., Matsushita, K., et al. 2012, PASJ, 64, 95

Simionescu, A., Werner, N., Böhringer, H., et al. 2009, A\&A, 493, 409

Smith, R. K., Brickhouse, N. S., Liedahl, D. A., \& Raymond, J. C. 2001, ApJ, 556, L91

Smith, R. J., Hudson, M. J., Nelan, J. E., et al. 2004, AJ, 128, 1558

Snowden, S. L., Egger, R., Freyberg, M. J., et al. 1997, ApJ, 485, 125

Snowden, S. L., Mushotzky, R. F., Kuntz, K. D., \& Davis, D. S. 2008, A\&A, 478,615

Springel, V., Frenk, C. S., \& White, S. D. M. 2006, Nature, 440, 1137

Sun, M. 2012, New J. Phys., 14, 045004

Sun, M., \& Vikhlinin, A. 2005, ApJ, 621, 718

Sun, M., Donahue, M., \& Voit, G. M. 2007, ApJ, 671, 190

Sun, M., Voit, G. M., Donahue, M., et al. 2009, ApJ, 693, 1142

Valentinuzzi, T., Poggianti, B. M., Fasano, G., et al. 2011, A\&A, 536, A34

Varela, J., D'Onofrio, M., Marmo, C., et al. 2009, A\&A, 497, 667

Vijayaraghavan, R., \& Ricker, P. M. 2015, MNRAS, 449, 2312

Vikhlinin, A., Markevitch, M., \& Murray, S. S. 2001, ApJ, 551, 160

Zhang, B., Sun, M., Ji, L., et al. 2013, ApJ, 777, 122 


\section{Appendix A: Optical data and kinematical analysis}

We analysed the Smith et al. (2004) galaxy sample, which is the largest available homogeneous sample in the field of view of the Hydra A cluster with galaxies with a measured line-of-sight (LOS) velocity $(V=c z)$. The sample contains 42 galaxies and the mean error of this sample is $\delta V \sim 25 \mathrm{~km} \mathrm{~s}^{-1}$.

For the centre of Hydra $\mathrm{A}$, we adopted the position of the brightest cluster galaxy (BCG) at $V_{\mathrm{BCG}}=16452$ $\mathrm{km} \mathrm{s}^{-1}\left[\mathrm{RA}=09^{\mathrm{h}} 18^{\mathrm{m}} 05^{\mathrm{s}} .69\right.$, Dec. $\left.=-12^{\circ} 05^{\prime} 43.8^{\prime \prime} \quad(\mathrm{J} 2000)\right]$. Following Girardi et al. (1998), we performed a two-step procedure to select cluster members. First, we selected the densest peak in the redshift distribution using the DEDICA reconstruction method (Pisani 1993). Second, we rejected other possible interlopers in the projected phase-space using the "shifting gapper" method (Fadda et al. 1996). With the first step, we detected Hydra $A$ as a peak of 36 galaxies at $z \sim 0.055$ (see Fig. 12), whereas the second step led us to reject three more galaxies. The resulting sample of 33 fiducial members is shown in Fig. A.1 (see also the inset of Fig. 12 in the Sect. 3.3). By applying the bi-weight estimator Beers et al. (1990) to the 33 cluster members of the whole system, we obtained a mean cluster redshift of $\langle z\rangle=0.0548 \pm 0.0004$, i.e. $\langle V\rangle=(16421 \pm 121) \mathrm{km} \mathrm{s}^{-1}$.

We computed the LOS velocity dispersion, $\sigma_{V}$, using the bi-weight estimator and applying the cosmological correction and standard correction for velocity errors (Danese et al. 1980). We obtained $\sigma_{V}=723_{-72}^{+93} \mathrm{~km} \mathrm{~s}^{-1}$, where errors are estimated through a bootstrap technique. This estimate is within the range of a few alternative estimates presented by Durret et al. (2009) and with that expected for clusters with X-ray temperatures $\mathrm{kT} \sim 3.5 \mathrm{keV}$ (Sato et al. 2012) in the assumption of the density-energy equipartition between ICM and galaxies. In fact, we estimated $\beta_{\text {spec }} \sim 1.05$, where $\beta_{\text {spec }}=\sigma_{V}^{2} /\left(k T / \mu m_{\mathrm{p}}\right)$ with $\mu=0.58$ the mean molecular weight and $m_{\mathrm{p}}$ the proton mass, and $\beta_{\text {spec }}=1$ is expected in the case of density-energy equipartition. Based on the value of $\sigma_{V}$, we estimated a mass $M_{200} \sim 4 \times 10^{14} h_{70}^{-1} M_{\odot}$ within a radius $R 200_{\text {opt }} \sim 1.5 h_{70}^{-1}$ Mpc (Munari et al. 2013).

The bright galaxy LEDA $87445\left(\mathrm{RA}=09^{\mathrm{h}} 18^{\mathrm{m}} 27^{\mathrm{s}} .47\right.$, Dec. $\left.=-12^{\circ} 22^{\prime} 23.9^{\prime \prime} \quad(\mathrm{J} 2000)\right)$ is a cluster member lying at the high velocity part of the cluster-galaxies velocity distribution $\left(V_{\mathrm{LEDA}}=17224 \mathrm{~km} \mathrm{~s}^{-1}\right)$. Inspection of Fig. A.1 suggests that regions around the BCG and LEDA 87445 are populated by galaxies with different kinematics. To investigate this point we applied the Dressler \& Schectman test (Dressler \& Shectman 1988, DStest). This is a powerful test for $3 \mathrm{D}$ substructure, which is sensitive in samples down to 30 member galaxies, at least in the case of important mergers (Pinkney et al. 1996). This test analyses the deviations of the local kinematics, as based on the i-th galaxy and its ten neighbours, from global kinematics. The DStest kinematical estimator is based on both mean velocity and velocity dispersion. For details, we refer to the original paper and to Barrena et al. (2011), in which two alternative kinematical estimators are presented. These estimators separately consider the contributions to the mean velocity and velocity dispersion (DS $\langle V\rangle$ and $\mathrm{DS} \sigma_{V}$-tests). We checked the significance of the existence of substructure by running 1000 Monte Carlo simulations, randomly shuffling the galaxy velocities. We detected a substructure with a c.l. of $99.4 \%$ (and $99.7 \%$ in the DS $\langle V\rangle$ case). Similar results $(99.1 \%$ and $99.7 \%)$ are obtained using local groups of six galaxies, corresponding to $N_{\text {local }}=\sqrt{N_{\text {gal }}}$ as suggested by (Bird 1994, see Fig. 13 in Sect. 3.3).

Following Girardi et al. (1996), we also searched for the presence of a velocity gradient by performing a multiple linear

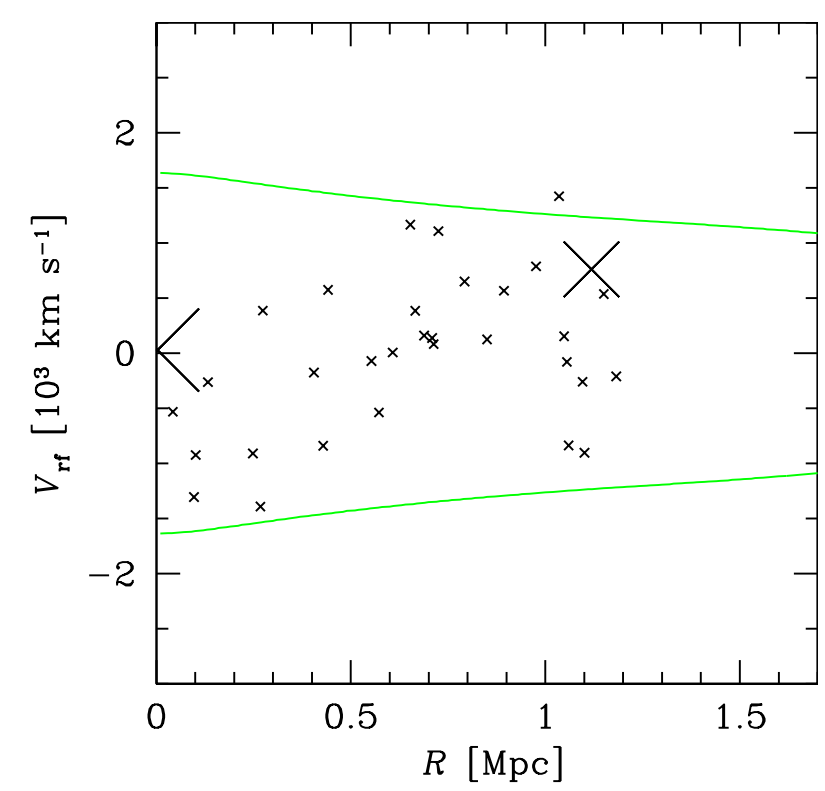

Fig. A.1. Rest-frame velocity $V_{\mathrm{rf}}=(V-\langle V\rangle) /(1+z)$ vs. projected clustercentric distance $R$ for the 33 cluster members. Crosses indicate the BGC (centre) and LEDA 87445. Curves contain the region of $V_{\mathrm{rf}}$ smaller than the escape velocity. The curves show the limit due to the escape velocity in the cluster.

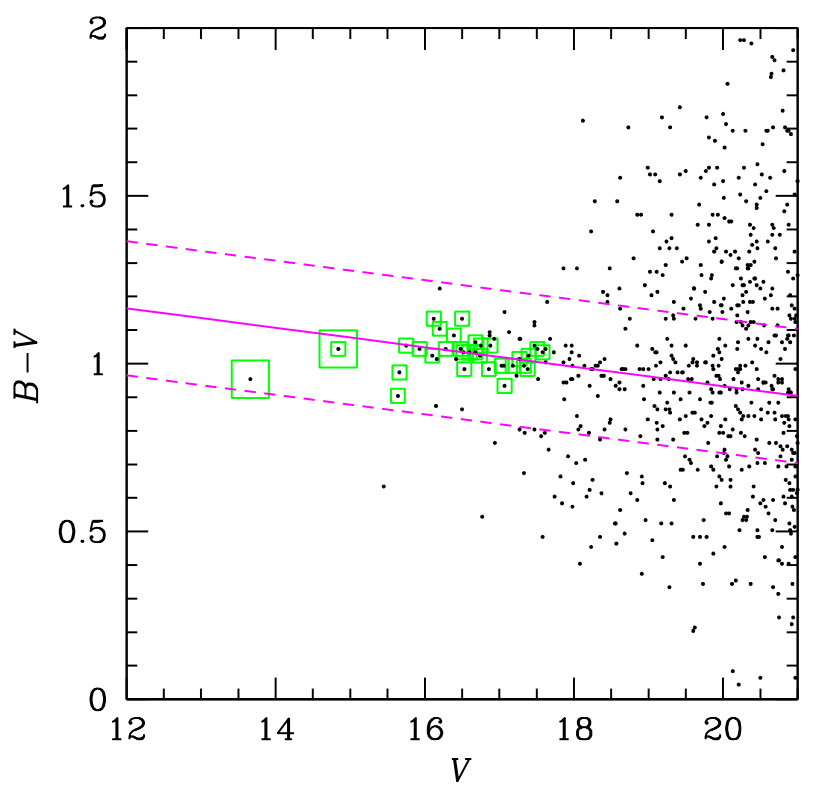

Fig. A.2. $B-V$ vs. $V$ diagram for galaxies in the photometric WINGS catalogue having $V<21$. Green squares indicate spectroscopic members and, in particular, the two large squares indicate the BGC and LEDA 87445. The solid line gives the best-fit CMR as determined on spectroscopic member galaxies; the dashed lines define the region within which photometric cluster members are selected.

regression fit to the observed velocities with respect to the galaxy positions in the plane of the sky. The significance is again based on 1000 Monte Carlo simulated clusters. We found only marginal evidence (at $90 \%$ c.l.) of the existence of a velocity gradient. The position angle PA $=143_{-21}^{+29}$ (measured counterclockwise from north) has a large error but agrees with Fig. 13 in detecting higher velocities in the S-SE region of Hydra A.

We also analysed the spatial distribution of the 33 spectroscopic member galaxies using the 2D adaptive-kernel method of Pisani (1996) (hereafter 2D-DEDICA). In addition to the central 


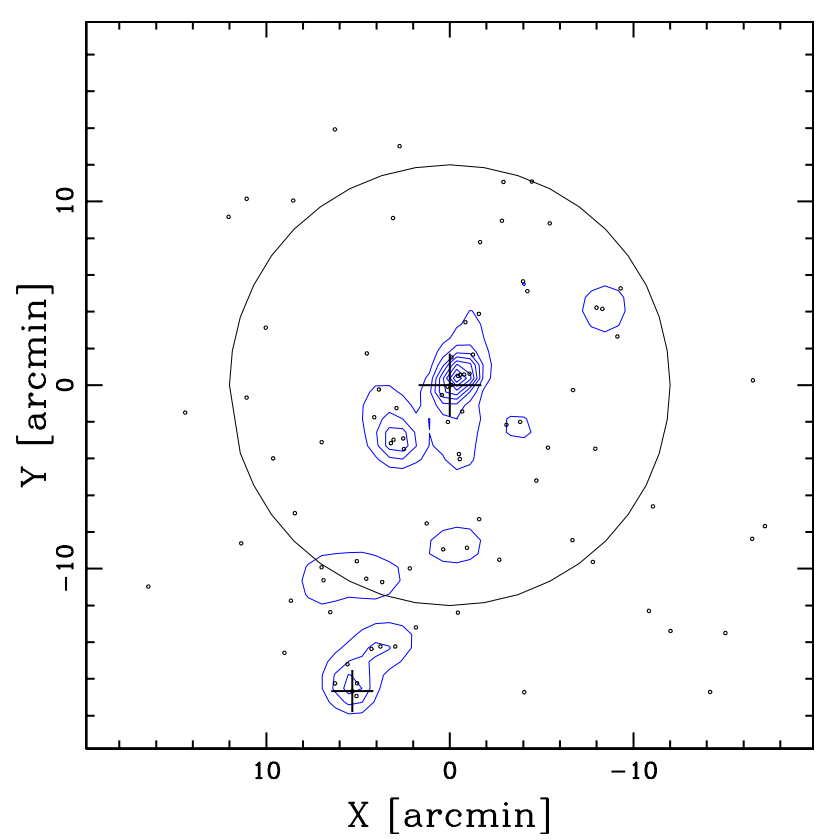

Fig. A.3. Spatial distribution on the sky of the 89 photometric likely cluster members with $V \leq 18.5$ (black small points). The contour map is obtained with the 2D-DEDICA method (blue lines). The larger and smaller crosses indicate the position of BCG and LEDA 87445. The plot is centred on the cluster centre and the circle contains the region within a radius equal to $12^{\prime}, \sim 0.5 R 200_{\text {opt }}$.

main density peak, we also detected a SSE peak coincident to LEDA 87445 , but only significant at the $98 \%$ c.l. In the study of the $2 \mathrm{D}$ galaxy distribution, the use of the spectroscopic sample is limited by both the poor number of galaxies and the possible incompleteness because of unavoidable constraints in the design of the fiber positioning during the spectroscopy. The analysis of the published WINGS ${ }^{2}$ photometric catalogue (Varela et al. 2009) offers an additional description of the 2D cluster structure. The WINGS catalogue has been based on deep $(B, V)$ widefield images $\left(\sim 35^{\prime} \times 35^{\prime}\right)$. It is $90 \%$ complete at $V \sim 21.7$ and the star/galaxy classification of objects $V<20$ was checked visually. In this study we focus on the red sequence galaxies, which are well-known good tracers of the cluster substructure (e.g. Lubin et al. 2000) and above all are useful to minimize the field contamination. We retrieved Vizier photometric data for 1632 WINGS galaxies in the Hydra A field from CDS. We corrected the Galactic extinction $A_{B}=0.148$ and $A_{V}=0.112$ following values listed by $\mathrm{NED}^{3}$. We selected photometric members on the basis of ( $B-V$ vs. $V$ ) colour-magnitude relation (CMR), in particular we used aperture magnitudes [MAG(10kpc)] for $B-V$ and total magnitude [MAG_AUTO] for $V$. To determine the CMR we applied a recursive $2 \sigma$-clipping fitting procedure to the spectroscopic member galaxies. During this procedure, since these members sample a small magnitude range, we fixed the slope equal to -0.047 , which is the median slope of WINGS clusters (Valentinuzzi et al. 2011). We selected a subsample of 24 members for which we fit the $\operatorname{OLS}(y / x)$ relation $B-V=1.513( \pm 0.171)-0.029( \pm 0.010) \times V$; the slope is consistent with that obtained for colours estimated in $5 \mathrm{kpc}$ (Valentinuzzi et al. 2011). In agreement with Valentinuzzi et al. (2011), we only considered galaxies within a colour range of 0.2 (see Fig. A.2). Moreover, we limited our analysis to galaxies with $V \leq 18.5$ (89 in the whole photometric catalogue), i.e. $\sim 3$ mag after $M^{*}$, to reduce the contamination of non-member galaxies. Figure A.3 shows the contour map, where we detected three density peaks significant at the $99 \%$ c.l., among which is a SSE peak at the location of the LEDA 87445 galaxy. This peak has a relative density $\rho_{\mathrm{S}}=0.4$ with respect to the central main peak, although the number of related members is very small (five galaxies).
2 http://web.oapd.inaf.it/wings/

3 NASA/IPAC Extragalactic Database which is operated by the Jet Propulsion Laboratory, California Institute of Technology, under contract with the NASA. 